\title{
Optimization of Wireless Relaying With Flexible UAV-Borne Reflecting Surfaces
}

\author{
Taniya Shafique, Hina Tabassum, and Ekram Hossain
}

\begin{abstract}
This paper presents a theoretical framework to analyze the performance of integrated unmanned aerial vehicle (UAV)-intelligent reflecting surface (IRS) relaying system in which IRS provides an additional degree of freedom combined with the flexible deployment of full-duplex UAV to enhance communication between ground nodes. Our framework considers three different transmission modes: (i) UAV-only mode, (ii) IRS-only mode, and (iii) integrated UAV-IRS mode to achieve spectral and energy-efficient relaying. For the proposed modes, we provide exact and approximate expressions for the end-to-end outage probability, ergodic capacity, and energy efficiency (EE) in closed-form. We use the derived expressions to optimize key system parameters such as the UAV altitude and the number of elements on the IRS considering different modes. We formulate the problems in the form of fractional programming (e.g. single ratio, sum of multiple ratios or maximization-minimization of ratios) and devise optimal algorithms using quadratic transformations. Furthermore, we derive an analytic criterion to optimally select different transmission modes to maximize ergodic capacity and EE for a given number of IRS elements. Numerical results validate the derived expressions with Monte-Carlo simulations and the proposed optimization algorithms with the solutions obtained through exhaustive search. Insights are drawn related to the different communication modes, optimal number of IRS elements, and optimal UAV height.
\end{abstract}

\section{Index Terms}

Unmanned aerial vehicle (UAV), intelligent reflecting surface (IRS), integrated UAV-IRS wireless communications, selection combining, outage probability, ergodic capacity, energy efficiency, fractional programming.

\section{INTRODUCTION}

Intelligent reflecting surfaces (IRS) are emerging as a key enabling technique to smartly reconfigure wireless propagation environment in beyond $5 \mathrm{G}$ wireless networks [1] . The IRS consists of multiple small

T. Shafique and E. Hossain are with the Department of Electrical and Computer Engineering, University of Manitoba, Canada. H. Tabassum is with the Department of Electrical Engineering and Computer Science, York University, Canada.The work was supported by a Discovery Grant from the Natural Sciences and Engineering Research Council of Canada (NSERC). 
meta-surfaces that are also referred to as IRS elements. IRS enables smart reconfiguration via softwarecontrolled reflections and is energy-efficient since meta-surfaces contain low-cost polymer diode/switch and conductive square patches [2] [3]. The comprehensive intelligent functionality of each element includes reflection, refraction, transmittance and absorption [1], [4]. The functionalities can be used all together or in separate based on the application requirement. In contrast to conventional relays that require active transmission and reception, the IRSs do not require any additional radio channel/frequency for signal transmission or reception which makes IRS cost-effective.

Unlike conventional IRS relaying, integrating IRS with the unmanned aerial vehicles (UAVs) allow flexible deployment of metasurfaces while minimizing the on-board UAV energy consumption [5], [6]. The proactive placement of integrated UAV-IRS system offers a cost-effective solution with minimal energy consumption and reduced network-wide spectrum resources. In this paper, we consider the mathematical performance characterization and optimization of an integrated UAV-IRS system.

\section{A. Background Work}

A series of research works $[7]-[[10]$ considered signal-to-noise ratio (SNR) outage characterization of UAV-assisted relaying assuming either line-of-sight (LoS) Rician or non-LoS (NLoS) Nakagami- $m$ faded aerial channels. The derived expressions are generally in the form of complicated mathematical functions that cannot be directly used for network planning and optimization purposes. For instance, [7], [8] provided closed-form expressions for the SNR outage probability assuming Nakagami- $m$ faded aerial channels with no notion of LoS and NLoS transmissions. In addition, the authors in [9] assumed Rician-faded LoS aerial channels and derived the SNR outage in the form of Marcum $Q$-function. In [10], the SNR outage probability was analyzed for Rician and Rayleigh fading channels considering LoS and NLoS channels, respectively. The aforementioned research works [7]-[10] overlooked the impact of limited on-board energy of the UAV as well as the circuit and hovering power consumption of the UAV.

Another series of research works that focused on the energy efficiency maximization of UAV-enabled relaying networks include [11] $-[19]$. These research works are solely based on numerical optimization techniques. Very recently, we developed a mathematical framework to characterize the reliability, energy efficiency, and coverage probability in a UAV-assisted data ferrying network considering Rician-faded aerial channels [18]. Using the derived expressions, we optimized the UAV data ferrying distance in three different problem settings, (i) minimize the energy consumption under the constraint of outage probability, (ii) minimize the outage probability under the constraint of energy consumption, and (iii) minimize both 
the outage probability and energy consumption by considering multi-objective optimization [18]. The aforementioned research works did not consider the IRS-assisted UAV systems.

To date, a number of research works considered the statistical performance characterization or optimization of IRS-assisted wireless networks either without UAV [2], [20]-[22] or with UAV [23]. A pioneering effort to characterize an upper bound on the average symbol error probability has been undertaken in [20]. The research work considered Rayleigh fading channels and simplified the instantaneous SNR given the optimal phase shifts for IRS. The energy efficiency of the system was not considered. A number of research works [2], [21] focused on maximizing the energy efficiency by optimizing the IRS phase shifts with infinite and low phase resolution capability. An interesting research work is [22] where the authors compared the performance of decode and forward (DF) relaying and IRS-assisted transmission. The IRS and DF relay were placed in the same fixed location. They also considered maximal ratio combining between the direct and IRS assisted link. Nevertheless, the channel gain coefficients were assumed to be perfectly known. The authors in [23] considered an IRS to facilitate the transmission between a mobile UAV and a ground user. The UAV-to-IRS transmission link was modeled as LoS Rician fading channel whereas IRS-to-ground user link was modeled as NLoS Rayleigh fading channel. The authors maximized the rate by optimizing IRS phase shifts and the trajectory through numerical optimization considering known channel state information (CSI). Finally, in [24], the authors optimized the location of the integrated IRS-UAV system using reinforcement learning approach.

\section{B. Paper Contribution and Organization}

Except [20], most of the aforementioned research works are focused on the optimization of the phaseshifts in IRS-assisted networks using numerical optimization techniques. Furthermore, the performance characterization and optimization of integrated UAV-IRS system have not been investigated yet.

This paper develops a comprehensive mathematical framework to characterize the performance of an integrated UAV-IRS system and optimize critical network parameters such as the number of IRS elements and UAV altitude to maximize the spectral and energy efficiency. Note that IRS micro-controller can perform the optimal switching of IRS elements out of all elements therefore optimal $N$ can be realized in practice. At this point, it is noteworthy that maximization of energy efficiency and optimization of the number of IRS elements $(N)$ in an integrated UAV-IRS system is crucial due to two reasons: (i) given the limited UAV size, the number of IRS elements that can be deployed on a UAV is limited ${ }^{1}$, and (ii)

\footnotetext{
${ }^{1}$ The size of one IRS element is typically in the range $\lambda / 10-\lambda / 5[3]$, where $\lambda$ denotes the wavelength of the transmitted wave. As such, this limitation becomes more evident in low frequencies.
} 
due to the power consumption associated with each IRS element. Although the power consumption of each IRS element is low, the overall power consumption may become significant for a large number of active IRS elements depending on the phase resolution power consumption $P_{r}(b)$, which depends on the number of bits assigned to resolve the phases in an IRS element. For instance, $P_{r}(b)=5 \mathrm{dBm}$ for 1-bit resolution and $P_{r}(b)=45 \mathrm{dBm}$ for infinite resolution [21]. The $P_{r}(b)$ depends on the operating frequency and the type of power amplifier [25].

- We characterize the outage probability, ergodic capacity, and energy efficiency in an integrated UAVIRS system (where IRS surface is mounted on the UAV) considering three different modes, (i) $U A V$-only mode, where the UAV performs relaying in full-duplex mode, (ii) IRS-only mode, where the IRS performs relaying which is implicitly a full-duplex transmission without self-interference, and (iii) Integrated UAV-IRS mode, where both the UAV and IRS perform relaying and the receiver uses selection combining (SC). The considered model captures the LoS air-to-ground (AtG) Rician fading channels and power consumption of UAV and IRS.

- We provide approximate expressions to increase the mathematical tractability of the proposed framework for system optimization purposes. That is, we incorporate the derived expressions (after some transformations to tractable mathematical forms) into the optimization problems. Numerical results validate the derived expressions with Monte-Carlo simulations.

- We formulate a variety of the optimization problems where objective functions have a fractional form for IRS-only mode and UAV-only modes, i.e. (i) maximize EE to optimize the number of IRS elements, (ii) maximize EE to optimize the height of the IRS, (iii) minimize IRS power consumption to optimize the the number of IRS element and transmission power subject to rate constraints, and (iv) maximize EE to optimize the height of the UAV. We solve the aforementioned problems and derive optimal solutions using quadratic transformation as a tool from fractional programming. Closed-form optimal solutions are provided, wherever applicable.

- We derive an analytic criterion to optimally select the UAV-only and IRS-only transmission modes to maximize the capacity and EE for a given number of IRS elements.

- Numerical results compare the proposed optimal solutions with the solutions obtained through exhaustive search. We note that, compared to the UAV-only mode, the IRS-only mode is energy efficient at lower altitudes with low to moderate number of active IRS elements, and for larger distances between the UAV and the source or destination.

The remainder of the paper is organized as follows. We describe the system model in Section III. In 


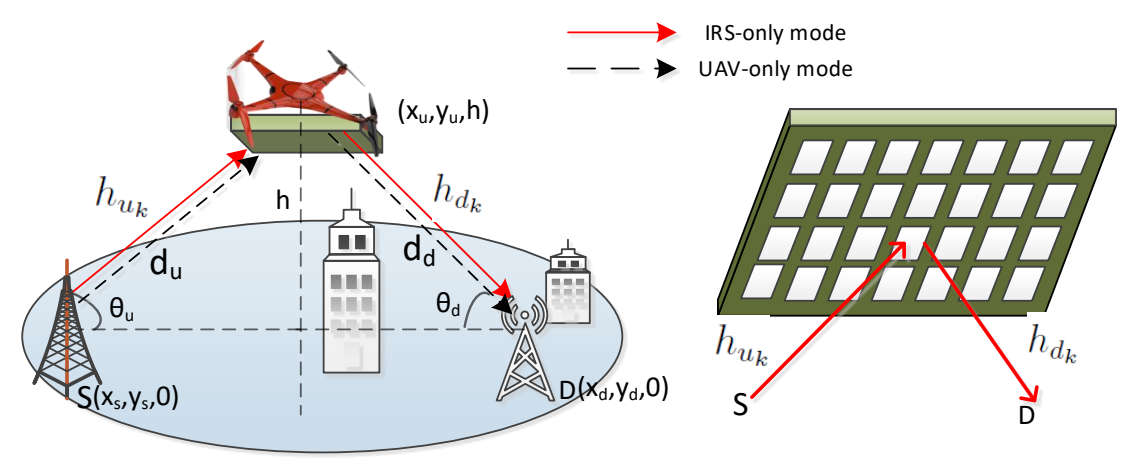

Fig. 1. Integrated UAV-IRS communication when IRS is placed on a UAV between the source and the destination.

Section III], we characterize the end-to-end energy efficiency, the SNR outage probability and data rate for the considered network modes. In Section IV, we propose approximations for erdogic capacity and energy efficiency. In Section V, optimization is performed to maximize energy efficiency for IRS elements and UAV height for transmission modes. Mode selection probability and criteria is proposed in the same section. Then, we present the numerical results in Section VI before we conclude in Section VII

\section{System Model AND Assumptions}

\section{A. Spatial Deployment of UAV-IRS system}

We consider an integrated UAV-IRS network in which a UAV carries a large array of IRS elements to assist communication between source $\mathbf{S}$ and destination $\mathbf{D}$ located on the ground. We assume that there exists no direct link between the S and D. In particular, the IRS reflects the incident signal in the desired direction of destination with minimal power consumption. In addition, the UAV operates as an independent relay between $\mathbf{S}$ and $\mathbf{D}$ since we assume that the UAV has separate transmit and receive antennas. In Cartesian coordinates, the locations of $\mathbf{S}$ and $\mathbf{D}$ are denoted as $\mathbf{w}_{s}=\left(x_{s}, y_{s}, 0\right)$, and $\mathbf{w}_{d}=\left(x_{d}, y_{d}, 0\right)$, respectively (Fig. 1). We also assume that UAV can be placed at any height $h$ such that $h \in\left[h_{\min }, h_{\max }\right]$ where $h_{\min }$ and $h_{\max }$ are decided by aviation authorities. We denote the UAV coordinate as $\mathbf{w}_{\mathbf{u}}=\left(x_{u}, y_{u}, h\right)$. In two-dimensional Cartesian coordinates, the location of source, destination, and the UAV can be given by $\mathbf{z}_{\mathbf{s}}=\left(x_{s}, y_{s}\right), \mathbf{z}_{\mathbf{d}}=\left(x_{d}, y_{d}\right)$, and $\mathbf{z}_{\mathbf{u}}=\left(x_{u}, y_{u}\right)$, respectively. 


\section{B. Aerial Channel Model}

The communication between the UAV and ground receiver $\mathbf{S}$ and $\mathbf{D}$ depends on the elevation angle between the nodes (and/or altitude of the UAV) and the environment (e.g. the intensity and heights of buildings). The transmission to the ground users may have LoS or non-LOS based on the elevation angle (in rad) between the UAV and $\mathrm{BS}_{i}$. The elevation angle can be given as follows:

$$
\theta_{u}=\arctan \left(\frac{h}{\left|\mathbf{z}_{\mathbf{u}}-\mathbf{z}_{\mathbf{s}}\right|}\right), \quad \theta_{d}=\arctan \left(\frac{h}{\left|\mathbf{z}_{\mathbf{u}}-\mathbf{z}_{\mathbf{d}}\right|}\right),
$$

where $h, \mathbf{z}_{\mathbf{s}}$, and $\mathbf{z}_{\mathbf{d}}$ are defined in II-A. The probability of LoS in each link is a function of $\theta_{i}$, i.e.

$$
p_{L}\left(\theta_{i}\right)=\left(1+e_{i} \exp \left(-g_{i}\left(\theta_{i}-e_{i}\right)\right)\right)^{-1}, \quad \forall i \in\{u, d\}
$$

where $e_{u}, e_{d}, g_{u}$, and $g_{d}$, are the environment parameters obtained from the curve fitting using Damped Least-Squares (DLS) method [26]. The path-loss exponent $\alpha$ is a function of the elevation angle [27], i.e.

$$
\alpha\left(\theta_{i}\right)=p_{L}\left(\theta_{i}\right) q_{i}+v_{i}
$$

Here $q_{u}, v_{u}, q_{d}$, and $v_{d}$ are constants depending on the uplink and downlink environment [26].

\section{Spectrum Allocation}

We consider that the destination $\mathrm{BS} \mathbf{D}$ has a data rate requirement $R_{0}$ which is defined as $R_{0}=$ $B \log _{2}\left(1+\Gamma_{0}\right)$. The $\Gamma_{0}$ represents the minimum end-to-end SNR threshold required by the destination to

achieve $R_{0}$, i.e. $\Gamma_{0}=2^{\frac{R_{0}}{B}}-1$. Here, $B$ represents the total transmission bandwidth available for IRS-only mode, UAV-only mode, and integrated UAV-IRS mode. The IRS does not need additional frequency to reflect the signals. For the sake of fairness, we consider in-band full-duplex (IBFD) operation for the UAV mode. This enables the UAV to transmit and receive simultaneously over the same frequency band $B$ which remains the same for all three modes of operation. The performance of IBFD communication is, however, limited by self interference (SI) which is introduced by the IBFD transmitter to its own receiver [28]. The antenna is assumed to be equipped with a three-port circulator to prevent the leakage of transmit chains to receive chains; however, in practice, perfect SI cancellation is not possible [29].

\section{Transmission Modes}

We consider three different modes of data transmission, i.e. (i) UAV-only mode, when UAV provides coverage to the destination D with all IRS elements switched off (absorbing state of IRS) and UAV is operating in IBFD transmission mode, (ii) IRS-only mode, when only IRS is responsible to provide service to the destination $\mathbf{D}$ by acting as relay and the UAV does not communicate, and (iii) Integrated 
UAV-IRS mode, when both IRS and UAV transmit the data and the receiver combines the data using selection combining ${ }^{2}$, i.e. by opportunistically selecting the stronger signal between those received from the UAV and the IRS. We consider that the receiver is equipped with a buffer to store the observations from IRS transmission that arrives one time slot prior to the UAV transmission. We consider that the IRS is equipped with uniform linear arrays of elements and there is a controller associated with IRS which is responsible for smart selection of the functionality of IRS elements such as absorption and beamforming.

Now we describe the transmission and channel models for each of the modes of operation.

1) UAV-only Mode: The transmission from $\mathbf{S}$ to UAV and the transmission from UAV to $\mathbf{D}$ can be given, respectively, as follows:

$$
y_{u}=\sqrt{\hat{A} p_{u} \eta_{u}^{-1} d_{u}^{-\alpha\left(\theta_{u}\right)}} h_{u} s+R_{\mathrm{SI}}+n_{u}, \quad y_{d}=\sqrt{\hat{A} p_{d} \eta_{d}^{-1} d_{d}^{-\alpha\left(\theta_{d}\right)}} h_{d} y_{u}+n_{d},
$$

where $s$ is the transmitted signal in binary phase shift keying (BPSK) from the source $\mathrm{S}$ to the UAV and $y_{u}$ is the signal received by the IBFD UAV and relayed to $\mathbf{D}, \eta_{i}$ denotes the excess aerial path-loss, $p_{u}$ is the transmission power of $\mathbf{S}$, and $p_{d}$ is the transmission power of UAV. Also, $d_{u}$ is the distance between the $\mathbf{S}$ and the UAV, i.e. $d_{u}=\sqrt{\left|\mathbf{z}_{u}-\mathbf{z}_{s}\right|^{2}+h^{2}}$ and $d_{d}$ is the distance between UAV and D, i.e. $d_{d}=\sqrt{\left|\mathbf{z}_{u}-\mathbf{z}_{d}\right|^{2}+h^{2}}$. Note that $\hat{A}$ reflects system parameters (e.g. operating frequency and antenna gain), $n_{i}$ is additive white Gaussian noise (AWGN) with zero-mean and power spectral density $N_{0}, R_{\mathrm{SI}}$ denotes the residual SI experienced by the UAV [28], and $h_{i}$ represents the $i$-th channel fading where $i \in(u, d)$. The SNR for the $i$-th is given as follows:

$$
\gamma_{i}=p_{i} \kappa_{i} d_{i}^{-\alpha\left(\theta_{i}\right)} X_{i}, \quad \forall i \in\{u, d\}
$$

where $\kappa_{u}=\frac{\hat{A} \eta_{u}^{-1}}{R_{\mathrm{SI}}+N_{0}}, \kappa_{d}=\frac{\hat{A} \eta_{d}^{-1}}{N_{0}}$, and $X_{i}=\left|h_{i}\right|^{2}$ follows non-central chi square distribution with mean $\Omega_{i}$, which is local mean power of $i$-th Rician fading channel whose probability density function (PDF) is:

$$
f_{X_{i}}(x)=\frac{K_{i}+1}{\Omega_{i}} e^{-K_{i}-\frac{\left(K_{i}+1\right) x}{\Omega_{i}}} I_{0}\left(2 \sqrt{\frac{K_{i}\left(K_{i}+1\right) x}{\Omega_{i}}}\right)=\sum_{\ell=0}^{\infty} \frac{b_{i}\left(b_{i} K_{i}\right)^{\ell}}{(\ell !)^{2}} x^{\ell} e^{-b_{i} x-K_{i}},
$$

in which $K_{i}$ is the Rician factor in the $i$-th link and $I_{0}$ is a modified Bessel function of the first kind and $b_{i}=\frac{K_{i}+1}{\Omega_{i}}[30]$. Note that in the UAV-only mode, the IRS absorbs the incoming signals to each element, and therefore, no information is relayed from IRS to the destination. We call this state as the non-active state of the IRS.

\footnotetext{
${ }^{2}$ In selection combining, the combiner outputs the signal on the branch with the highest SNR, which requires one receiver switching to active branch, and co-phasing of multiple branches is not required as is the case the other combining techniques. Therefore, selection combining exhibits low overhead, has a simplest receiver implementation, and is mathematically tractable.
} 
Assuming that the UAV can perform decoding of $y_{u}$ and then relay the decoded data, using (4), the end-to-end SNR $\Gamma_{\mathrm{UAV}}$ from $\mathbf{S}$ to $\mathbf{D}$ can be modeled as [31]:

$$
\Gamma_{\mathrm{UAV}}=\min \left\{\gamma_{u}, \gamma_{d}\right\}
$$

2) IRS-only Mode: In this mode, we assume that the UAV does not transmit and the IRS controller adjusts the phase shift of each element intelligently to the optimal value [20], [32]. That is, the IRS maximizes the signal power by optimizing the phase shifts of the impinging signals. In this setup, for the sake of symmetry, we consider the odd number of elements, i.e. $N=2 n+1$, where $n$ is any arbitrary positive integer. The received signal at destination $\mathbf{D}$ via $k$-th IRS element is given by

$$
y_{\mathrm{IRS}_{\mathrm{k}}}=\sqrt{\hat{A} p_{u} \eta_{u}^{-1} d_{u_{k}}^{-\alpha\left(\theta_{u_{k}}\right)}} h_{u_{k}} e^{j\left(\phi_{k}\right)} \sqrt{\hat{A} \eta_{d}^{-1} d_{d_{k}}^{-\alpha\left(\theta_{d_{k}}\right)}} h_{d_{k}} s+w_{u},
$$

where $k \in\{-n,-n+1, \cdots, 0,1, \cdots, n\}$. The distance between $j$-th element to $\mathbf{S}$ and to $\mathbf{D}$ can be given as $d_{u_{k}}=\sqrt{\left|\mathbf{z}_{s}-\mathbf{z}_{u_{k}}\right|^{2}+h^{2}}$ and $d_{d_{k}}=\sqrt{\left|\mathbf{z}_{d}-\mathbf{z}_{u_{k}}\right|^{2}+h^{2}}$, respectively, where $\mathbf{z}_{u_{k}}=\left(x_{u_{k}}, y_{u_{k}}\right)$ and $x_{u_{k}}=x_{u}-k D_{\text {IRS }}, y_{u_{k}}=y_{u}$, and $D_{\text {IRS }}$ denotes the uniform spacing between two consecutive elements on IRS. Note that the $k=0$-th element is at UAV location $\mathbf{z}_{u}$. The channel from $\mathbf{S}$ to $k$-th IRS element and $k$-th IRS element to $\mathbf{D}$ can be given as $h_{u_{k}}=\left|h_{u_{k}}\right| e^{-j \theta_{u_{k}}}$ and $h_{d_{k}}=\left|h_{d_{k}}\right| e^{-j \theta_{d_{k}}}$, respectively. The end-to-end SNR for IRS-only mode $\Gamma_{\text {IRS }}[20]$ for an IRS with $N$ elements can be given as follows:

$$
\Gamma_{\text {IRS }}=V\left(\sum_{k=1}^{N} d_{u_{k}}^{-\alpha\left(\theta_{u_{k}}\right) / 2} d_{d_{k}}^{-\alpha\left(\theta_{d_{k}}\right) / 2}\left|h_{u_{k}}\right|\left|h_{d_{k}}\right| e^{-j\left(\theta_{u_{k}}+\theta_{d_{k}}-\phi_{k}\right)}\right)^{2},
$$

where $V=\hat{A}^{2} p_{u} \eta_{u}^{-1} \eta_{d}^{-1} / N_{0}$. It is evident from (7) that the maximum SNR is obtained by taking the channel phases as $\phi_{k}-\theta_{u_{k}}-\theta_{d_{k}}=0, \forall k \in(-n,-n+1, \cdots, n-1, n)$ which maximizes the exponential term to unity [20]. Now the modified maximum SNR is given as follows:

$$
\Gamma_{\text {IRS }}=V\left(\sum_{k=1}^{N} d_{u_{k}}^{-\frac{\alpha\left(\theta_{u_{k}}\right)}{2}} d_{d_{k}}^{-\frac{\alpha\left(\theta_{d_{k}}\right)}{2}}\left|h_{u_{k}}\right|\left|h_{d_{k}}\right|\right)^{2} .
$$

Given the limited size of UAV and the IRS, we assume that the distance between $\mathbf{S}$ and $k$-th IRS element is approximately the same as the distance between $\mathbf{S}$ and UAV. Similarly, we assume that the distance between $\mathbf{D}$ and $k$-th IRS element is approximately the same as the distance between $\mathbf{D}$ and UAV. That is, $d_{u_{k}} \approx d_{u}, d_{d_{k}} \approx d_{d}, \theta_{u_{k}} \approx \theta_{u}$, and $\theta_{d_{k}} \approx \theta_{d}$. From this point onward, we will use $\alpha_{u}$ and $\alpha_{d}$ as $\alpha\left(\theta_{u_{k}}\right)$, and $\alpha\left(\theta_{d_{k}}\right)$, respectively, for brevity. Subsequently, (8) simplifies as follows:

$$
\Gamma_{\text {IRS }} \approx V d_{u}^{-\alpha_{u}} d_{d}^{-\alpha_{d}}\left(\sum_{k=1}^{N}\left|h_{u_{k}}\right|\left|h_{d_{k}}\right|\right)^{2} .
$$

Eq. (8) and its approximation in (9) are validated in Fig. 2 for different simulation parameters. Note that, the IRS implicitly operates in full-duplex mode (with zero self-interference) and the incident signals on IRS reflect with minimal delay (typically less than the decoding delay experienced in DF relaying). 
3) Integrated UAV-IRS Mode: Here, both the UAV and the IRS relay the signal transmitted from $\mathbf{S}$ and the receiver uses SC to extract the desired signal. The SNR at the receiver can be formulated as follows:

$$
\Gamma_{\mathrm{INT}}=\max \left(\Gamma_{\mathrm{UAV}}, \Gamma_{\mathrm{IRS}}\right)=\max \left\{\min \left(p_{u} \kappa_{u} d_{u}^{-\alpha_{u}} X_{u}, p_{d} \kappa_{d} d_{d}^{-\alpha_{d}} X_{d}\right), V \frac{\left(\sum_{k=1}^{N}\left|h_{u_{k}}\right|\left|h_{d_{k}}\right|\right)^{2}}{d_{u}^{\alpha_{u}} d_{d}^{\alpha_{d}}}\right\} .
$$

\section{E. Energy Consumption Model}

We consider that the UAV hovering time is equal to the time UAV can communicate and can be computed as $T_{\text {hov }}=\frac{E_{B}}{p_{\text {uav }}}$, where $E_{B}$ is maximum UAV battery capacity and $p_{\text {uav }}$ is the power consumption of the UAV. The total power consumption of the considered system includes (i) the power consumed by the UAV for hovering and supporting IRS transmissions $\left(p_{\text {uav }}\right)$ and data transmission $\left(p_{d}\right)$ in downlink and (ii) the hardware power consumption $\left(p_{b s}\right)$ of the ground BS transmitter and receiver as well as $\left(p_{u}\right)$.

1) UAV Power Consumption ( $\left.p_{\text {uav }}\right)$ : The total UAV power consumption is the sum of powers consumed by UAV in hovering $p_{h}$, circuit power consumption $p_{c}$ [33], and the power consumed by UAV in the IRS

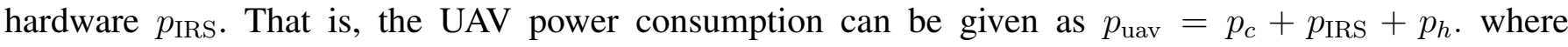

$p_{h}=\frac{\delta}{8} \rho s A \xi^{3} r^{3}+(1+\kappa) \sqrt{\frac{(m g)^{3}}{2 \rho A}}$, in which $\rho, A, \xi, r, s, \delta$, and $\kappa$ denote the air density (in $\mathrm{kg} / \mathrm{m}^{3}$ ), rotor disc area (in $\mathrm{m}^{2}$ ), blade angular velocity (in $\mathrm{rad} / \mathrm{sec}$ ), rotor radius (in $\mathrm{m}$ ), rotor solidity, profile drag coefficient, and incremental correction factor of induced power, respectively.

Since we consider that the IRS is mounted on a UAV, IRS power consumption is a part of the total UAV power consumption. Note that, IRS is acting as a passive device and does not need any transmission power. However, its power consumption is due to the number of IRS elements and the phase resolution [2] and is thus written as $p_{\mathrm{IRS}}=N P_{r}(b)$, where $P_{r}(b)$ is phase resolution power consumption. For instance, the power consumption of finite phase resolution for 6 bits is $P_{r}(6)=78 \mathrm{~mW}$ and for infinite phase resolution is $P_{r}(\infty)=45 \mathrm{dBm}$ (Fig. 4 of [21]). Therefore, an increase in the resolution and the number of IRS elements increases its hardware power consumption as formulated in [2], [21].

2) Terrestrial Circuit Power Consumption $\left(p_{b s}\right)$ : It is the hardware power consumption, i.e. the circuit power consumed by the source and destination ground BSs [34] given as $p_{b s}$.

3) Transmission Power Consumption: The transmission power consumption includes transmission power of the source BS in the uplink $\left(p_{u}\right)$ and that of the UAV in the downlink $\left(p_{d}\right)$.

Subsequently, we can define the total power consumption of each transmission mode as $P_{\mathrm{UAV}}=$ $p_{u}+p_{d}+C, P_{\mathrm{IRS}}=p_{u}+p_{\mathrm{IRS}}+C$, and $P_{\mathrm{INT}}=p_{u}+p_{d}+p_{\mathrm{IRS}}+C$, where $C=p_{c}+p_{h}+2 p_{\mathrm{bs}}$. 


\section{Performance Characterization of Integrated UAV-IRS Relaying}

In this section, we characterize the outage probability $O_{m}$, ergodic capacity $C_{m}$ and energy-efficiency $\mathrm{EE}_{\mathrm{m}}$ for each of the modes (i.e. UAV-only, IRS-only, and integrated UAV-IRS modes) for the considered integrated UAV-IRS relaying system. The subscript $m$ denotes the mode of operation.

\section{A. UAV-only Mode of Relaying}

Conditioned on the distances $d_{u}$ and $d_{d}$, the end-to-end SNR $\Gamma_{\mathrm{UAV}}$ can be given using (6). Subsequently, the SNR outage probability can be defined as follows:

$$
O_{\mathrm{UAV}}=\mathbb{P}\left(\Gamma_{\mathrm{UAV}}<\Gamma_{0}\right)=\mathbb{P}\left[\min \left(\gamma_{u}, \gamma_{d}\right)<\Gamma_{0}\right]=1-\left(1-F_{\gamma_{u}}\left(\Gamma_{0}\right)\right)\left(1-F_{\gamma_{d}}\left(\Gamma_{0}\right)\right),
$$

where $F_{\gamma_{u}}\left(\Gamma_{0}\right)$ and $F_{\gamma_{d}}\left(\Gamma_{0}\right)$ represent the CDFs of the SNR received on the channel from $\mathbf{S}$ to UAV and UAV to D, respectively, evaluated at the desired SNR threshold $\Gamma_{0}$. Using (4), the $i$-th link SNR outage can be given as follows:

$$
F_{\gamma_{i}}\left(\Gamma_{0}\right)=\mathbb{P}\left(\gamma_{i} \leq \Gamma_{0}\right)=\mathbb{P}\left(X_{i} \leq \Gamma_{i}^{\prime} d_{i}^{-\alpha_{i}}\right)=F_{X_{i}}\left(\Gamma_{i}^{\prime} d_{i}^{-\alpha_{i}}\right), \quad i \in\{u, d\}
$$

where $X_{i}=\left|h_{i}\right|^{2}$ represents non-central chi square distribution and $\Gamma_{i}^{\prime}=\frac{\Gamma_{0}}{\kappa_{i} p_{i}}$. Using the alternate exact expression for PDF in (5), the CDF of $X_{i}$ can be given as follows [30], [35], [36]:

$$
F_{X_{i}}\left(x_{i}\right)=1-\sum_{\ell=0}^{\infty} \sum_{m=0}^{\ell} f_{i}(m, l) x_{i}^{m} e^{-b x_{i}}
$$

where $f_{i}(m, \ell)=e^{-K_{i}} \frac{K_{i}^{\ell} b_{i}^{m}}{\ell ! m !}, b_{i}=\frac{K_{i}+1}{\Omega_{i}}, \Omega_{i}$ is the mean local power of the Rician channel in the $i$-th link, and $K_{i}$ is the Rician factor. Substituting $x_{i}=\Gamma_{i}^{\prime} d_{i}^{\alpha_{i}}$ in (13), we obtain

$$
F_{\gamma_{i}}\left(\Gamma_{0}\right)=F_{X_{i}}\left(\Gamma_{i}^{\prime} d_{i}^{\alpha_{i}}\right)=1-\sum_{\ell=0}^{\infty} \sum_{m=0}^{\ell} f_{i}(m, l)\left(\Gamma_{i}^{\prime} d_{i}^{\alpha_{i}}\right)^{m} \exp \left(-b_{i} \Gamma_{i}^{\prime} d_{i}^{\alpha_{i}}\right), \quad i \in(u, d) .
$$

By using (14) for $i=u$ and $i=d$ in (11), the end-to-end SNR outage $O_{\mathrm{UAV}}$ is given as

$$
O_{\mathrm{UAV}}=1-\sum_{\ell=0}^{\infty} \sum_{m=0}^{\ell} f_{u}(m, l)\left(\Gamma_{u}^{\prime} d_{u}^{\alpha_{u}}\right)^{m} \exp \left(-b_{u} \Gamma_{u}^{\prime} d_{u}^{\alpha_{u}}\right) \sum_{\ell=0}^{\infty} \sum_{m=0}^{\ell} f_{d}(m, l)\left(\Gamma_{d}^{\prime} d_{d}^{\alpha_{d}}\right)^{m} \exp \left(-b_{d} \Gamma_{d}^{\prime} d_{d}^{\alpha_{d}}\right) .
$$

Corollary 1. In scenarios where NLoS components are dominant (i.e. for $K_{u}=0$ and $K_{d}=0$ ) than LoS components, the Rician distribution follows Rayleigh distribution. As such, the CDF in (14) can be expressed as $F_{X_{u}}\left(\Gamma_{u}^{\prime} d_{u}^{-\alpha_{u}}\right)=1-e^{-\Gamma_{u}^{\prime} d_{u}^{-\alpha_{u}} / \Omega_{u}}, F_{X_{d}}\left(\Gamma_{d}^{\prime} d_{d}^{-\alpha_{d}}\right)=1-e^{-\frac{\Gamma_{d}^{\prime}}{\Omega_{d}} d_{d}^{\alpha_{d}}}$. The end-to-end SNR outage for UAV-only mode of relaying can be simplified as follows:

$$
O_{\mathrm{UAV}}\left(d_{d}\right)=1-e^{-\frac{\Gamma_{u}^{\prime}}{\Omega_{u}} d_{u}^{-\alpha_{u}}-\frac{\Gamma_{d}^{\prime}}{\Omega_{d}} d_{d}^{-\alpha_{d}}} .
$$




\section{B. Outage Probability for IRS-only Mode of Relaying}

Using (9), the end-to-end SNR outage in the IRS-only mode of relaying can be given as:

$$
O_{\mathrm{IRS}}=\mathbb{P}\left(\Gamma_{\mathrm{IRS}} \leq \Gamma_{0}\right)=\mathbb{P}\left(V d_{u}^{-\alpha_{u}} d_{d}^{-\alpha_{d}} Z^{2} \leq \Gamma_{0}\right)=\mathbb{P}\left(Z^{2} \leq t \Gamma_{0}\right)
$$

where $Z=\sum_{k=1}^{N}\left|h_{u_{k}}\right|\left|h_{d_{k}}\right|$ and $t=\frac{d_{u}^{\alpha u} d_{d}^{\alpha}}{V}$. The SNR outage probability can then be derived as follows.

Proposition 1. The outage probability of IRS-only mode can be given as follows:

$$
O_{\mathrm{IRS}}=\frac{1}{2}\left(\operatorname{erf}\left(\frac{\sqrt{t \Gamma_{0}}-\sqrt{\lambda}}{\sqrt{2}}\right)+\operatorname{erf}\left(\frac{\sqrt{t \Gamma_{0}}+\sqrt{\lambda}}{\sqrt{2}}\right)\right)
$$

where $t=\frac{d_{u}^{\alpha u} d_{d}^{\alpha} d}{V}, \lambda=\frac{1}{2} \frac{\mu_{Z}^{2}}{\sigma_{Z}^{2}}, \mu_{z}=(N+1) \mathbb{E}\left[\left|h_{u_{k}}\right|\left|h_{d_{k}}\right|\right]$, and $\sigma_{Z}^{2}=(N+1) \operatorname{var}\left(\left|h_{u_{k}}\right|\left|h_{d_{k}}\right|\right)$.

Proof. In general, a meta-surface is made up of a large number of reflecting elements, i.e. $N \gg 1$. Therefore, we apply central limit theorem (CLT) on $Z=\sum_{k=1}^{N}\left|h_{u_{k}}\right|\left|h_{d_{k}}\right|$, where $\left|h_{u_{k}}\right|$ and $\left|h_{d_{k}}\right|$ are identically and independently distributed (i.i.d) random variables with the mean and variance $\mathbb{E}\left[\left|h_{i_{k}}\right|\right]$ and $\operatorname{var}\left(\left|h_{i_{k}}\right|\right)$, respectively, for $i \in(u, d)$. Subsequently, the distribution of $Z$ will converge to the Gaussian distribution with mean and variance, respectively, given by

$$
\mu_{z}=(N+1) \mathbb{E}\left[\left|h_{u_{k}}\right|\left|h_{d_{k}}\right|\right] \quad \text { and } \quad \sigma_{Z}^{2}=(N+1) \operatorname{var}\left(\left|h_{u_{k}}\right|\left|h_{d_{k}}\right|\right) .
$$

Note that $\left|h_{u_{k}}\right|$ and $\left|h_{d_{k}}\right|$ are independent, but may not be identically distributed Rician variables. Therefore, we consider that the product $\left|h_{u_{k}}\right|\left|h_{d_{k}}\right|$ is the product of two independent but non-identical Rician random variables. The product follows the double-Rician distribution [37] with the mean and variance given as:

$$
\mathbb{E}\left[\left|h_{u_{k}}\right|\left|h_{d_{k}}\right|\right]=\sigma \frac{\pi}{2}{ }_{1} F_{1}\left(\frac{-1}{2} ; 1 ; \frac{-\mu_{u}^{2}}{2 \Omega_{u}}\right){ }_{1} F_{1}\left(\frac{-1}{2} ; 1 ; \frac{-\mu_{d}^{2}}{2 \Omega_{d}}\right),
$$

and

$$
\operatorname{var}\left(\left|h_{u_{k}}\right|\left|h_{d_{k}}\right|\right)=2^{2} \sigma^{2}\left(1+\frac{\mu_{u}^{2}}{2 \Omega_{u}}\right)\left(1+\frac{\mu_{d}^{2}}{2 \Omega_{d}}\right)-\left(\sigma \frac{\pi}{2}\right)^{2}\left[{ }_{1} F_{1}\left(\frac{-1}{2} ; 1 ; \frac{-\mu_{u}^{2}}{2 \Omega_{u}}\right){ }_{1} F_{1}\left(\frac{-1}{2} ; 1 ; \frac{-\mu_{d}^{2}}{2 \Omega_{d}}\right)\right]^{2},
$$

where $\sigma^{2}=\Omega_{u} \Omega_{d}$ and ${ }_{1} F_{1}($.$) is the Confluent Hypergeometric function. Now taking X=Z^{2}$, the distribution of $X$ follows the non-central chi square distribution with unity degree of freedom and noncentrality parameter $\lambda=\frac{1}{2} \frac{\mu_{Z}^{2}}{\sigma_{Z}^{2}}$. Subsequently, the probability density function (PDF) of $X$ is given as:

$$
f_{X}(x)=\frac{1}{2}\left(\frac{x}{\lambda}\right)^{-1 / 4} e^{-\frac{\lambda+x}{2}} I_{-1 / 2}(\sqrt{\lambda x}),
$$

where $I_{\beta}$ is the modified Bessel function of first kind of order $\beta$. Fig. 3 shows that the PDF of $X$ obtained from simulations converges to non-central chi square variable for $N \geq 20$, as is implied by CLT.

$$
O_{\text {IRS }}=\mathbb{P}\left(Z^{2} \leq t \Gamma_{0}\right)=\mathbb{P}\left(-\sqrt{t \Gamma_{0}} \leq X \leq \sqrt{t \Gamma_{0}}\right)=\int_{x=0}^{t \Gamma_{0}} \frac{1}{2}\left(\frac{X}{\lambda}\right)^{-1 / 4} e^{-\frac{\lambda+X}{2}} I_{-1 / 2}(\sqrt{\lambda X}) d X .
$$



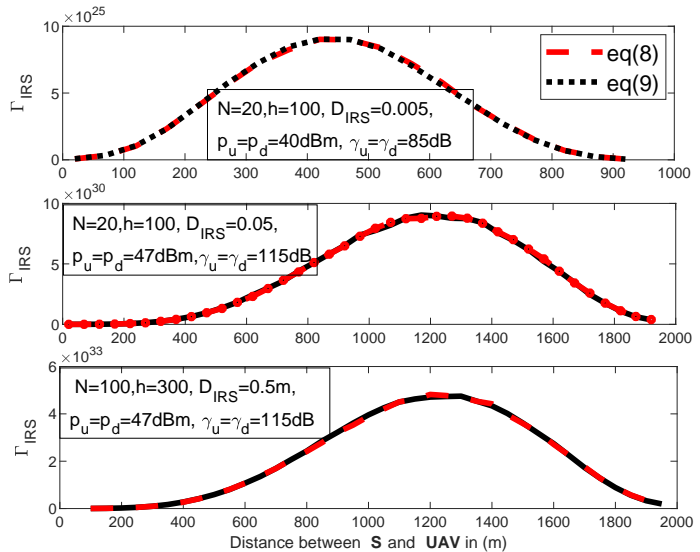

Fig. 2. Comparison of $(8)$ and its approximation in 97 for different parameters.

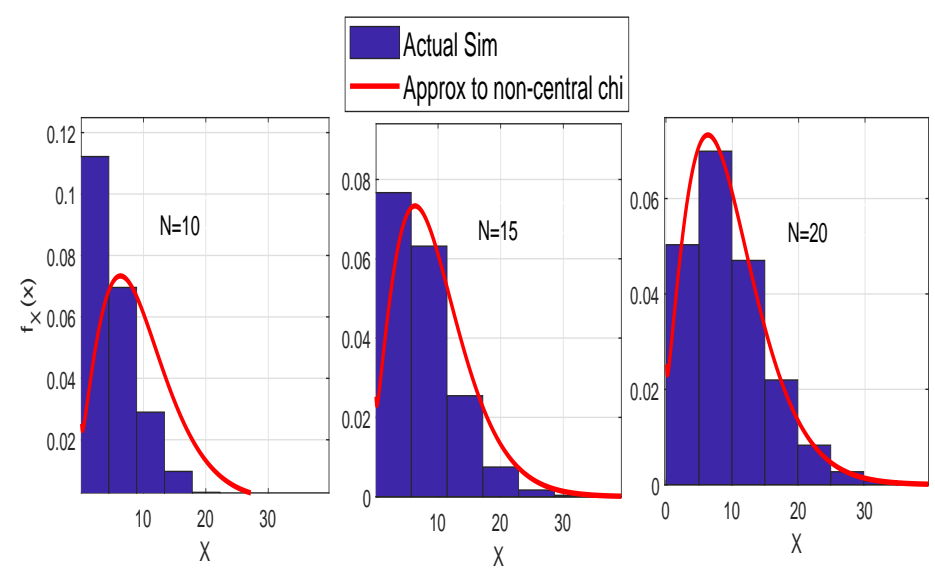

Fig. 3. Comparison of the exact PDF of $X$ through simulations and the PDF of $X$ when $N \rightarrow \infty$ that follows non-central chi-square distribution.

For $\mu_{u}=\mu_{d}=0$, the mean and variance of the double Rician variable can be simplified as follows.

Corollary 2. For $\mu_{u}=\mu_{d}=0$, the double Rician variable converts to double Rayleigh variable. Thus, the mean and variance of the product $\left|h_{u_{k}}\right|\left|h_{d_{k}}\right|$ can be simplified as $\mathbb{E}\left[\left|h_{u_{k}}\right|\left|h_{d_{k}}\right|\right]=\sigma \frac{\pi}{2}$ and var $\left(\left|h_{u_{k}}\right|\left|h_{d_{k}}\right|\right)=$ $2^{2} \sigma^{2}\left(1-\pi^{2} / 16\right)$ with $\sigma^{2}=\Omega_{u} \Omega_{d}[38]$, respectively. After applying central limit theorem for Rayleigh fading, we obtain $\mu_{z}=(N+1) \sigma \frac{\pi}{2}, \sigma_{Z}^{2}=(N+1) 2^{2} \sigma^{2}\left(1-\pi^{2} / 16\right)$.

\section{Outage Probability of Integrated UAV-IRS Mode of Relaying}

In integrated UAV-IRS mode of relaying, we assume that the receiver applies SC and selects the mode of operation associated to the maximum SNR. This implies additional degree of freedom, however, at the expense of increased resource consumption, since both the UAV and the IRS are actively transmitting to D. The outage probability of this mode can thus be derived using (10) as follows:

$$
O_{\mathrm{INT}}=\operatorname{Pr}\left(\Gamma_{\mathrm{UAV}} \leq \Gamma_{0}\right) \operatorname{Pr}\left(\Gamma_{\mathrm{IRS}} \leq \Gamma_{0}\right)=O_{\mathrm{UAV}} O_{\mathrm{IRS}},
$$

where $O_{\mathrm{UAV}}$ and $O_{\mathrm{IRS}}$ are given in Section III.A and III.B, respectively.

\section{Ergodic Capacity $C_{m}$ and Energy Efficiency $\mathrm{EE}_{m}$ for Mode $m$}

Given mode $m$, the exact end-to-end ergodic capacity at the receiver can be derived as follows [39]:

$$
C_{m}=\mathbb{E}\left[B \log _{2}\left(1+\Gamma_{m}\right)\right]=\frac{1}{\ln (2)} \int_{t=0}^{\infty} \frac{\operatorname{Pr}\left(\Gamma_{m}>t\right)}{1+t} d t=\frac{B}{\ln (2)} \int_{\Gamma_{0}=0}^{\infty} \frac{1-O_{m}}{1+\Gamma_{0}} d \Gamma_{0}
$$


where $\operatorname{Pr}\left(\Gamma_{m}>\Gamma_{0}\right)=1-O_{m}$ and $O_{m}$ is derived in (15), (18), and (21) for UAV-only, IRS-only and integrated UAV-IRS modes, respectively. Along the similar lines, using the definition of energy efficiency $\mathrm{EE}_{m}$ of each mode $m$ (which is defined as the ratio of ergodic capacity to the corresponding power consumption $P_{m}$ ), we can derive the exact end-to-end energy-efficiency as follows:

$$
\mathrm{EE}_{m}=\frac{B}{\ln (2) \mathrm{P}_{\mathrm{m}}} \int_{\Gamma_{0}=0}^{\infty} \frac{1-O_{m}}{1+\Gamma_{0}} d \Gamma_{0} .
$$

\section{Approximate Performance Characterizations For UAV-IRS RELAying}

In this section, we first derive a bound on the ergodic capacity $C_{m}$ and energy efficiency $\mathrm{EE}_{\mathrm{m}}$ for each mode of relaying (i.e. UAV-only, IRS-only, and UAV-IRS modes).

UAV-only Mode of Relaying: Applying Jensen's Inequality to the ergodic capacity expression, an upper bound on the ergodic capacity (in bps) can be derived as follows:

$$
\mathbb{E}\left[\log _{2}\left(1+\mathrm{SNR}_{\mathrm{m}}\right)\right] \leq \log _{2}\left(1+\mathbb{E}\left[\mathrm{SNR}_{\mathrm{m}}\right]\right)
$$

Subsequently, we derive tractable expressions of the ergodic capacity and energy-efficiency in UAV-only mode as shown in the following Proposition.

Proposition 2. The ergodic capacity $C_{\mathrm{UAV}}$ and energy-efficiency E $E_{\mathrm{UAV}}$ expressions in UAV-only mode can be given, respectively, as follows:

$$
\begin{gathered}
C_{\mathrm{UAV}} \leq B \log _{2}\left(1+\min \left(p_{u} \kappa_{u} d_{u}^{-\alpha_{u}} \Omega_{u}, p_{d} \kappa_{d} d_{d}^{-\alpha_{d}} \Omega_{d}\right)\right) \\
\mathrm{EE}_{\mathrm{UAV}} \approx \frac{B \log _{2}\left(1+\min \left(p_{u} \kappa_{u} d_{u}^{-\alpha_{u}} \Omega_{u}, p_{d} \kappa_{d} d_{d}^{-\alpha_{d}} \Omega_{d}\right)\right)}{p_{u}+p_{d}+C} .
\end{gathered}
$$

Proof. The ergodic capacity $C_{\mathrm{UAV}}$ in 22 can be bounded as follows:

$$
\begin{aligned}
& C_{\mathrm{UAV}} \stackrel{(a)}{\leq} B \log _{2}\left(1+\mathbb{E}\left[\min \left(p_{u} \kappa_{u} d_{u}^{-\alpha_{u}} X_{u}, p_{d} \kappa_{d} d_{d}^{-\alpha_{d}} X_{d}\right)\right]\right) \\
& \stackrel{(b)}{\approx} B \log _{2}\left(1+\min \left(\mathbb{E}\left[p_{u} \kappa_{u} d_{u}^{-\alpha_{u}} \quad X_{u}, p_{d} \kappa_{d} d_{d}^{-\alpha_{d}} X_{d}\right]\right)\right) \\
& \stackrel{(c)}{=} B \log _{2}\left(1+\min \left(p_{u} \kappa_{u} d_{u}^{-\alpha_{u}} \mathbb{E}\left[X_{u}\right], p_{d} \kappa_{d} d_{d}^{-\alpha_{d}} \mathbb{E}\left[X_{d}\right]\right)\right),
\end{aligned}
$$

where $X_{u}$ and $X_{d}$ follow non-central chi square distribution. Note that (a) is obtained by using Jensen's inequality [40], (b) is obtained by interchanging $\min ($.$) and \mathbb{E}($.$) (validated in Fig. 4), (c) follows from$ non-central chi-square distribution with mean $\Omega_{u}$ and $\Omega_{d}$, respectively, and results in (25). Finally, using ergodic capacity in (25), we obtain $\mathrm{EE}_{\mathrm{UAV}}$ in $(26)$.

Fig. 4 validates the accuracy of our proposed bounds in (27)(step a) using Jensen's inequality and 27)(step b) using interchange of $\min (\cdot)$ and the expectation operator $\mathbb{E}[\cdot]$ with exact Monte-Carlo simulations. To further justify the approximation in (b), we calculate the expectation of the minimum of 
two random variables, i.e. $\mathbb{E}\left[\Gamma_{\mathrm{UAV}}\right]=\mathbb{E}\left[\min \left(p_{u} \kappa_{u} d_{u}^{-\alpha_{u}} X_{u}, p_{d} \kappa_{d} d_{d}^{-\alpha_{d}} X_{d}\right)\right]$ in an exact form. That is, we first determine the PDF of $\Gamma_{\mathrm{UAV}}$, by taking the derivative of the CDF of $\Gamma_{\mathrm{UAV}}$. The CDF of $\Gamma_{\mathrm{UAV}}$ can be derived using (15), by substituting $\Gamma_{u}^{\prime}$ and $\Gamma_{d}^{\prime}$ and replacing $\Gamma_{0}$ with $z$. Finally, we calculate $\mathbb{E}\left[\Gamma_{\mathrm{UAV}}\right]=\int_{z=0}^{\infty} z f_{\Gamma_{\mathrm{UAV}}}(z) d z$, under the condition that $m_{u}+m_{d}$ and $m_{u}+m_{d}>0$ and $\left(\frac{b_{u} d_{u}^{\alpha_{u}}}{\kappa_{u} p_{u}}+\frac{b_{d} d_{d}^{\alpha_{d}}}{\kappa_{d} p_{d}}\right) \geq 0$.

Corollary 3. When NLoS components are dominant, the Rician distribution follows Rayleigh distribution, i.e. $\Omega_{u}=1$ and $\Omega_{d}=1$. The end-to-end $\mathrm{EE}_{\mathrm{UAV}}$ for UAV-only mode in (26) can be simplified as follows:

$$
\mathrm{EE}_{\mathrm{UAV}} \approx \frac{\operatorname{Blog}_{2}\left(1+\min \left(\mathrm{p}_{\mathrm{u}} \kappa_{\mathrm{u}} \mathrm{d}_{\mathrm{u}}^{-\alpha_{\mathrm{u}}}, \mathrm{p}_{\mathrm{d}} \kappa_{\mathrm{d}} \mathrm{d}_{\mathrm{d}}^{-\alpha_{\mathrm{d}}}\right)\right)}{\mathrm{p}_{\mathrm{u}}+\mathrm{p}_{\mathrm{d}}+\mathrm{C}} .
$$

IRS-only Mode of Relaying: For IRS-only mode, the ergodic capacity and EE expressions are derived in the following.

Proposition 3. The ergodic capacity expression can be obtained for IRS-only mode as follows:

$$
C_{\mathrm{IRS}} \stackrel{(a)}{\leq} B \log _{2}\left(1+\mathbb{E}\left[V d_{u}^{-\alpha_{u}} d_{d}^{-\alpha_{d}} X\right]\right) \stackrel{(b)}{=} B \log _{2}\left(1+V d_{u}^{-\alpha_{u}} d_{d}^{-\alpha_{d}}(v+\lambda)\right),
$$

where (a) is obtained using Jensen's inequality and $(b)$ is obtained using $\mathbb{E}[X]=\nu+\lambda[41]$. Using (29)(step b), we bound $\mathrm{EE}_{\mathrm{IRS}}$ as follows:

$$
\mathrm{EE}_{\mathrm{IRS}} \approx \frac{\operatorname{Blog}_{2}\left(1+\mathrm{Vd}_{\mathrm{u}}^{-\alpha_{\mathrm{u}}} \mathrm{d}_{\mathrm{d}}^{-\alpha_{\mathrm{d}}}(\nu+\lambda)\right)}{\mathrm{P}_{\mathrm{IRS}}}
$$

Integrated UAV-IRS Mode of Relaying: For integrated UAV-IRS mode ( $m=$ INT), the ergodic capacity in (22) can be bounded as:

$$
C_{\mathrm{INT}}=B \mathbb{E}\left[\log _{2}\left(1+\max \left(V d_{u}^{-\alpha_{u}} d_{d}^{-\alpha_{d}} X, \min \left(p_{u} \kappa_{u} d_{u}^{-\alpha_{u}} X_{u}, p_{d} \kappa_{d} d_{d}^{-\alpha_{d}} X_{d}\right)\right)\right)\right],
$$

where $X, X_{u}$, and $X_{d}$ follow non-central chi square distribution representing end-to-end channel fading power in IRS transmission, channel fading power from $\mathbf{S}$ to UAV and UAV to $\mathbf{D}$, respectively. After applying SC, the ergodic capacity (31) can be approximated as follows:

$$
\begin{aligned}
C_{\mathrm{INT}} & \stackrel{(a)}{\leq} B \log _{2}\left(1+\mathbb{E}\left[\max \left(V d_{u}^{-\alpha_{u}} d_{d}^{-\alpha_{d}} X, \min \left(p_{u} \kappa_{u} d_{u}^{-\alpha_{u}} X_{u}, p_{d} \kappa_{d} d_{d}^{-\alpha_{d}} X_{d}\right)\right)\right]\right) \\
& \stackrel{(b)}{\approx} B \log _{2}\left(1+\max \left(V d_{u}^{-\alpha_{u}} d_{d}^{-\alpha_{d}} \mathbb{E}[X], \mathbb{E}\left[\min \left(\hat{A} p_{u} \eta_{u}^{-1} d_{u}^{-\alpha_{u}} X_{u}, \hat{A} p_{d} \eta_{d}^{-1} d_{d}^{-\alpha_{d}} X_{d}\right)\right]\right)\right) \\
& \stackrel{(c)}{\approx} B \log _{2}\left(1+\max \left(V d_{u}^{-\alpha_{u}} d_{d}^{-\alpha_{d}} \mathbb{E}[X], \min \left(p_{u} \kappa_{u} d_{u}^{-\alpha_{u}} \mathbb{E}\left[X_{u}\right], p_{d} \kappa_{d} d_{d}^{-\alpha_{d}} \mathbb{E}\left[X_{d}\right]\right)\right)\right) \\
& \stackrel{(d)}{=} B \log _{2}\left(1+\max \left(V d_{u}^{-\alpha_{u}} d_{d}^{-\alpha_{d}}(\nu+\lambda), \min \left(p_{u} \kappa_{u} d_{u}^{-\alpha_{u}} \Omega_{u}, p_{d} \kappa_{d} d_{d}^{-\alpha_{d}} \Omega_{d}\right)\right)\right),
\end{aligned}
$$

where (a) is obtained using Jensen's inequality, (b) and (c) are obtained by interchanging $\max ($.$) and$ $\min ($.$) operators with the \mathbb{E}($.$) operator, respectively, and (d) is obtained by substituting the mean of X$, $X_{u}$ and $X_{d}$ with $\nu+\lambda, \Omega_{u}$ and $\Omega_{d}$, respectively. Finally, using (d) we approximate $\mathrm{EE}_{\mathrm{INT}}$ as follows:

$$
\mathrm{EE}_{\mathrm{INT}} \approx \frac{\operatorname{Blog}_{2}\left(1+\max \left(\operatorname{Vd}_{\mathrm{u}}^{-\alpha_{\mathrm{u}}} \mathrm{d}_{\mathrm{d}}^{-\alpha_{\mathrm{d}}}(\nu+\lambda), \min \left(\mathrm{p}_{\mathrm{u}} \kappa_{\mathrm{u}} \mathrm{d}_{\mathrm{u}}^{-\alpha_{\mathrm{u}}} \Omega_{\mathrm{u}}, \mathrm{p}_{\mathrm{d}} \kappa_{\mathrm{d}} \mathrm{d}_{\mathrm{d}}^{-\alpha_{\mathrm{d}}} \Omega_{\mathrm{d}}\right)\right)\right)}{\mathrm{P}_{\mathrm{INT}}} .
$$




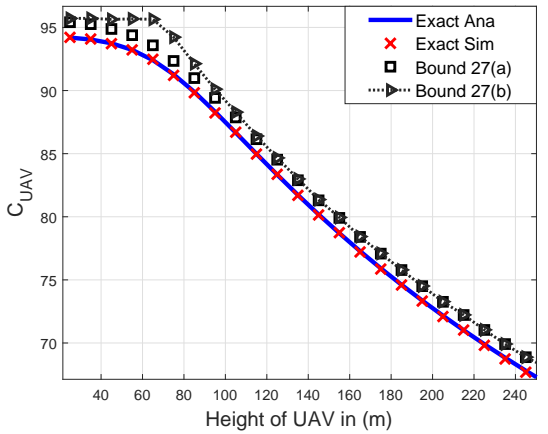

Fig. 4. The comparison of exact ergodic capacity (via analysis and simulations) to the UAV-only bounds provided in Eq. 27(step a) and Eq. 27(step b). Approximation in Eq. 27 (step b) is validated by analytically solving Eq. 27(step a).

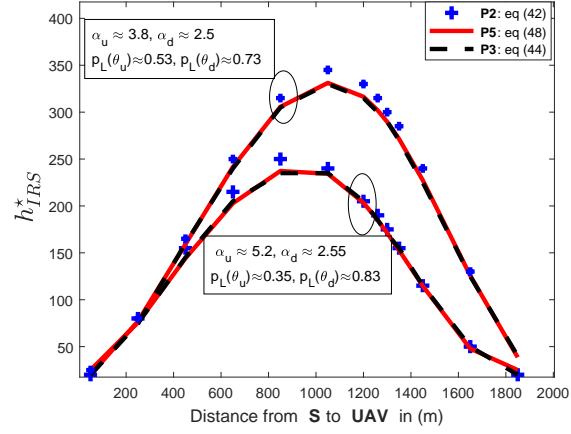

Fig. 5. The validation of solution obtained from (42), (44) and (48) for IRS-only mode for $E_{b} / N_{0}=130 \mathrm{~dB}, R_{\mathrm{SI}}=38 \mathrm{~dB}$, $N=30, p_{u}=p_{d}=50 \mathrm{dBm}$ for different environment parameters, where $\left(E_{b}\right.$ : per symbol energy).

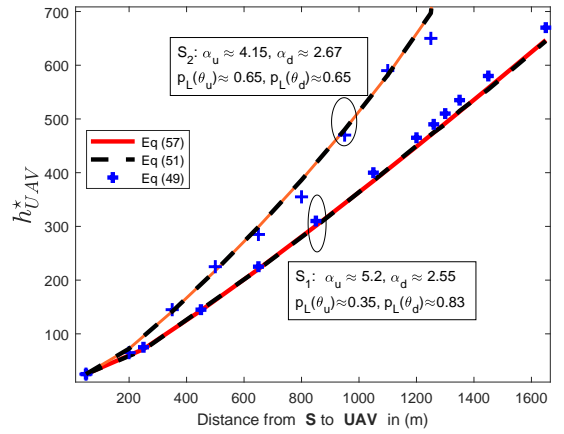

Fig. 6. The validation of solution obtained from (49), 51) and (57) for UAV-only mode for $E_{b} / N_{0}=135 \mathrm{~dB}, R_{\mathrm{SI}}=50 \mathrm{~dB}$, $p_{u}=p_{d}=45 \mathrm{dBm}$ for different environment parameters. For given parameters $I_{i}<10^{10}$ $\forall i \in(u, d)$ assures the concavity of $O_{i}(h)$.

\section{OPTIMIZATION OF UAV-IRS RELAYING}

In this section, we consider two optimization problems for maximizing the network energy efficiency and minimizing the network power consumption subject to rate constraints, considering the UAV-only mode and the IRS-only mode of relaying. For the IRS-only mode, we optimize the number of active IRS elements $N$ and height of the IRS surface (i.e. UAV height). For the UAV-only mode, we optimize the UAV height.

\section{A. IRS-only Mode: Optimizing the Number of IRS Elements}

1) EE Maximization: Using (30) where $\lambda$ is a function of $N$, i.e. $\lambda=(N+1) \lambda^{\prime}$, where $\lambda^{\prime}=$ $\frac{1}{2} \frac{\left(\mathbb{E}\left[\left|h_{u_{k}}\right|\left|h_{d_{k}}\right|\right]\right)^{2}}{\operatorname{var}\left(\left|h_{u_{k}}\right|\left|h_{d_{k}}\right|\right)}$ can be taken from (18), the EE maximization problem can be formulated as follows:

$$
\begin{aligned}
& \mathbf{P 1}: \max _{N} \mathrm{EE}_{\mathrm{IRS}}=\frac{\mathrm{B} \log _{2}\left(1+\mathrm{Vd}_{\mathrm{u}}^{-\alpha_{\mathrm{u}}} \mathrm{d}_{\mathrm{d}}^{-\alpha_{\mathrm{d}}}(\mathrm{v}+\lambda)\right)}{\mathrm{p}_{\mathrm{u}}+\mathrm{NP}_{\mathrm{r}}(\mathrm{b})+\mathrm{C}} \\
& \text { s.t. } \mathbf{C 1}: N_{\min } \leq N \leq N_{\max },
\end{aligned}
$$

where $N_{\max }$ is the maximum number of IRS elements that can be calculated as a ratio of the size of UAV to the size of one IRS element, $N_{\min }$ is the minimum number of IRS elements that can be deployed at a surface in practical settings and for which the objective function is accurate [refer to Fig. 3]. Since $\lambda$ is directly proportional to $N$, we reformulate the problem P1 as follows:

$$
\begin{aligned}
& \mathbf{P 2}: \max _{\lambda} \frac{\operatorname{Blog}_{2}\left(1+\mathrm{Vd}_{\mathrm{u}}^{-\alpha_{\mathrm{u}}} \mathrm{d}_{\mathrm{d}}^{-\alpha_{\mathrm{d}}}(\mathrm{v}+\lambda)\right)}{\mathrm{p}_{\mathrm{u}}+\left(\frac{\lambda-\lambda^{\prime}}{\lambda^{\prime}}\right) \mathrm{P}_{\mathrm{r}}(\mathrm{b})+\mathrm{C}} \\
& \text { s.t. } \mathbf{C 1}:\left(N_{\min }+1\right) \lambda^{\prime} \leq \lambda \leq\left(N_{\max }+1\right) \lambda^{\prime} .
\end{aligned}
$$


The problem $\mathrm{P} 2$ is non-convex in general; however, it is in the form of ratio of concave and convex function w.r.t variable $\lambda$. Fortunately, due to the structure of the problem, the global optimal solution can be obtained by applying quadratic transform proposed in [42]. The quadratic transform converts the ratio of concave and convex function to the convex form by introducing an auxiliary variable $y$. Thus, we optimize the primal variable $\lambda$ and the auxiliary variable $y_{j}$ at each iteration $j$.

The iterative algorithm is guaranteed to converge to the global optimal solution for the single ratio objective function in P1. As such, using Quadratic Transform, the problem $\mathbf{P 2}$ can be reformulated as:

$$
\text { P3 : } \max _{\lambda, y} Q(\lambda)=2 y \sqrt{B \log _{2}\left(1+V d_{u}^{-\alpha_{u}} d_{d}^{-\alpha_{d}}(v+\lambda)\right)}-y^{2}\left(p_{u}+\left(\frac{\lambda-\lambda^{\prime}}{\lambda^{\prime}}\right) P_{r}(b)+C\right)
$$

s.t. C1.

For a given $\lambda$, in each iteration $j, y_{j}^{*}$ can be found in closed-form as $y_{j}^{\star}=\frac{\sqrt{B \log _{2}\left(1+V d_{u}^{-\alpha u} d_{d}^{-\alpha} d(v+\lambda)\right)}}{p_{u}+\left(\frac{\lambda-\lambda^{\prime}}{\lambda^{\prime}}\right) P_{r}(b)+C}$. Now we solve P3 using Algorithm 1 for which the convergence to the global optimal solution is proved in [43].

\section{Algorithm 1: Optimization of Number of IRS Elements in IRS-only Mode}

Data: Initialize $\lambda, j=1$, Maximum Iterations $J_{\max }$, Error tolerance $\epsilon, Q\left(\lambda^{j}\right)$

Find $y_{j}^{\star}$ by solving $Q\left(\lambda^{j}\right)$ and set $j=2$;

while $\left|y_{j-1}^{\star}-y_{j}^{\star}\right| \geq \epsilon$ and $j<J_{\max }$ do

- Update $\lambda$ by solving $Q\left(\lambda^{j}\right)$ for fixed $y_{j-1}^{\star}$ using any convex optimization tool, e.g. CVX.

- update $y_{j}^{\star}$

- $j=j+1$.

Result: optimal desired solution $N^{\star}$ is then obtained from $\lambda^{\star}$ using $N^{\star}=\frac{\lambda^{\star}-\lambda^{\prime}}{\lambda^{\prime}}$,

2) Minimization of Power Consumption Under Rate Constraint: The problem can be formulated as:

$$
\begin{aligned}
& \mathbf{P 1}: \min _{N} p_{u}+N P_{r}(b)+C \\
& \text { s.t. } \mathbf{C} 1: B \log _{2}\left(1+V d_{u}^{-\alpha_{u}} d_{d}^{-\alpha_{d}}\left(v+(N+1) \lambda^{\prime}\right)\right) \geq R_{0} \\
& \quad \mathbf{C 2}: N_{\min } \leq N \leq N_{\max } .
\end{aligned}
$$

The objective function in $\mathbf{P 1}$ is convex and monotonically decreasing w.r.t $N$ and the IRS ergodic capacity is monotonically increasing function of $N$. Therefore, the solution to the optimization problem lies at the boundary of the constraint $\mathbf{C 1}$, which is given as follows:

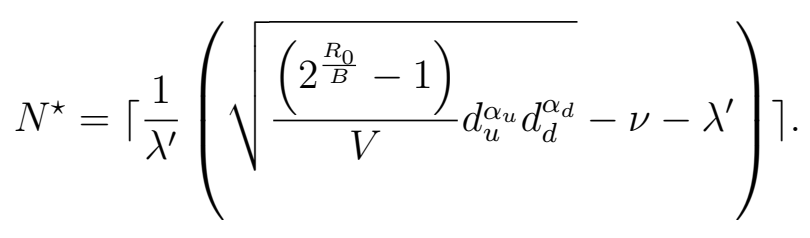


The generalized optimal solution $N^{\star}$ is provided by incorporating the bound $\mathbf{C 2}$ as follows:

$$
N^{\star}= \begin{cases}N_{\min } & N^{\star} \leq N_{\min } \\ N_{\max } & N^{\star} \geq N_{\max } . \\ \left\lceil\frac { 1 } { \lambda ^ { \prime } } \left(\sqrt{\left.\left.\frac{\left(2^{\frac{R_{0}}{B}}-1\right)}{V} d_{u}^{\alpha_{u}} d_{d}^{\alpha_{d}}-\nu-\lambda^{\prime}\right)\right\rceil}\right.\right. & \text { otherwise }\end{cases}
$$

In addition, the optimization problem (37) can be solved to optimize the variable $p_{u}$ given a fixed $N$. The objective function in $\mathbf{P 1}$ is convex and monotonically increasing w.r.t $p_{u}$, whereas the ergodic capacity is monotonically increasing function on $p_{u}$. Therefore, the solution to the optimization problem lies at the boundary of the constraint $\mathbf{C 1}$ and the optimal solution for $p_{u}^{\star}$ can be given as follows:

$$
p_{u}^{\star}=\frac{2^{\frac{R_{0}}{B}}-1}{\hat{A}^{2} \eta_{u}^{-1} \eta_{d}^{-1}} \frac{d_{u}^{\alpha_{u}} d_{d}^{\alpha_{d}} N_{0}}{v+(N+1) \lambda^{\prime}} .
$$

\section{B. IRS-only Mode: Height Optimization}

Here, we maximize $\mathrm{EE}_{\mathrm{IRS}}$ which is equivalent to maximizing the ergodic capacity $C_{\mathrm{IRS}}$ in 29) w.r.t height, since the IRS-only power consumption does not depend on height. The problem can then be formulated as follows:

$$
\begin{aligned}
& \mathbf{P 1}: \max _{h} \mathrm{C}_{\mathrm{IRS}}=B \log _{2}\left(1+V d_{u}^{-\alpha_{u}} d_{d}^{-\alpha_{d}}(\nu+\lambda)\right) \\
& \text { s.t. } \mathbf{C} 1: h_{\min } \leq h \leq h_{\max } .
\end{aligned}
$$

In (41), we note that only numerator $d_{u}^{-\alpha_{u}} d_{d}^{-\alpha_{d}}$ is a function of $h$. Therefore, to reformulate $\mathbf{P 1}$ we ignore the logarithm and constants in the objective function of $\mathbf{P 2}$ as shown below:

$$
\begin{aligned}
& \mathbf{P} 2: \max _{h} d_{u}^{-\alpha_{u}} d_{d}^{-\alpha_{d}} \\
& \text { s.t. } \mathbf{C 1}
\end{aligned}
$$

The optimal $h$ obtained from $\mathbf{P 2}$ can be substituted back in (41) to obtain maximum EE $\mathrm{IRS}_{\text {. By combining }}$ (1), (2), and (3), we note that $\alpha_{u}=\frac{q_{u}}{1+\varsigma_{u} \exp \left(-g_{u} \arctan \left(\frac{h}{z_{u}}\right)\right)}+v_{u}$ is a function of $h$, where $\varsigma_{u}=e_{u} e^{g_{u} e_{u}}$ and $\hat{z}_{u}=\left|\mathbf{z}_{\mathbf{u}}-\mathbf{z}_{\mathbf{S}}\right|$. Similarly, $\alpha_{d}=\frac{q_{d}}{1+\varsigma_{d} \exp \left(-g_{d} \arctan \left(\frac{h}{\hat{z}_{d}}\right)\right)}+v_{d}$ is a function of $h$, where $\varsigma_{d}=e_{d} e^{g_{d} e_{d}}$ and $\hat{z}_{d}=\left|\mathbf{z}_{\mathbf{u}}-\mathbf{z}_{\mathbf{d}}\right|$. Clearly, the reformulated objective function in $\mathbf{P 2}$ depends on $\alpha_{i}, i \in\{u, d\}$ which is non-linear due to tangent inverse function of variable $h$ in the denominator of $\alpha_{u}$ and $\alpha_{d}$.

Subsequently, we apply the following transformations to simplify the problem:

- Taking the $\log$ of objective function of $\mathbf{P 2}$, the transformed objective function becomes

$$
-\alpha_{u} \log \left(d_{u}\right)-\alpha_{d} \log \left(d_{d}\right)=-\frac{\alpha_{u}}{2} \log \left(\hat{z}_{u}^{2}+h^{2}\right)-\frac{\alpha_{d}}{2} \log \left(\hat{z}_{d}^{2}+h^{2}\right) .
$$


- Using $\arctan (x) \approx \frac{3 x}{1+2 \sqrt{1+x^{2}}}$, we get

$$
\alpha_{i} \approx q_{i}\left(1+\varsigma_{i} \exp \left(\frac{-3 g_{i} h}{\hat{z}_{i}+2 \sqrt{\hat{z}_{i}^{2}+h^{2}}}\right)\right)^{-1}+v_{i}, \quad i \in\{u, d\}
$$

- Applying the second-order Taylor series approximation $\exp (-x) \approx 1-x+\frac{x^{2}}{2}$ and some algebraic manipulations, we obtain

$$
\alpha_{i}(h) \approx \frac{A_{i}\left(\hat{z}_{i}+2 \sqrt{\hat{z}_{i}^{2}+h^{2}}\right)^{2}-B_{i} h\left(\hat{z}_{i}+2 \sqrt{\hat{z}_{i}^{2}+h^{2}}\right)+C_{i} h^{2}}{\left(1+\varsigma_{i}\right)\left(\hat{z}_{i}+2 \sqrt{\hat{z}_{i}^{2}+h^{2}}\right)^{2}-B_{i}^{\prime} h\left(\hat{z}_{i}+2 \sqrt{\hat{z}_{i}^{2}+h^{2}}\right)+C_{i}^{\prime} h^{2}}, \quad i \in\{u, d\},
$$

where $A_{i}=q_{i}+v_{i}\left(1+\varsigma_{i}\right), B_{i}=3 \varsigma_{i} v_{i} g_{i}, C_{i}=9 / 2 v_{i} \varsigma_{i} g_{i}^{2}, B_{i}^{\prime}=3 \varsigma_{i} g_{i}, C_{i}^{\prime}=9 / 2 \varsigma_{i} g_{i}^{2}$. Following the above approximations, the original (42) is given as follows:

$$
\text { P3 : } \max _{h}-\frac{1}{2} \alpha_{u}(h) \log \left(\hat{z}_{u}^{2}+h^{2}\right)-\frac{1}{2} \alpha_{d}(h) \log \left(\hat{z}_{d}^{2}+h^{2}\right)
$$

$$
\text { s.t. C1. }
$$

The mismatch in the optimal solutions is found to be negligibly small and is mainly due to the considered arctan and Taylor approximations, as validated in Fig. 5. Clearly, the problem in P3 is in the form of sum of ratio of concave-convex function as is shown in the following Proposition. This guarantees that an optimal solution for $\mathbf{P 3}$ can be obtained.

Proposition 4. The $-\frac{1}{2} \alpha_{i}(h) \log \left(\hat{z}_{i}^{2}+h^{2}\right)$ is ratio of concave-convex when

$$
\hat{z}_{i}>10 \quad \& \quad\left\{\begin{array}{ll}
\hat{z}_{i} \geq h^{5 / 4}\left(\frac{78 A_{i}+14 C_{i}}{11 B_{i}}\right)^{1 / 4} & \hat{z}_{i} \geq h \\
h \geq \hat{z}_{i}\left(\frac{\left(78 A_{i} \hat{z}_{i}+B_{i}+14 C_{i} \hat{z}_{i}\right)}{12 B_{i}}\right)^{1 / 4} & h>\hat{z}_{i}
\end{array} .\right.
$$

\section{Proof. See Appendix A.}

Now, $\mathbf{P 3}$ can be reformulated as follows:

$$
\mathbf{P} 3^{\prime}: \min _{h} \frac{1}{2} \alpha_{u}(h) \log \left(\hat{z}_{u}^{2}+h^{2}\right)+\frac{1}{2} \alpha_{d}(h) \log \left(\hat{z}_{d}^{2}+h^{2}\right)
$$

\section{s.t. C1.}

Note that $\mathbf{P 3}$ is a multiple-ratio fractional programming problem and can be solved by applying the quadratic transform method, as applied earlier. For the sake of simplicity, we rewrite $\mathbf{P 3}^{\prime}$ by using a general notation $i$, where $i=(u, d)$, as follows [42]:

$$
\mathbf{P} 4: \min _{h} \sum_{i=(u, d)} \frac{O_{i}(h)}{R_{i}(h)}
$$

$$
\text { s.t. C1, }
$$


where $O_{i}(h)=0.5 \log \left(h^{2}+\hat{z}_{i}^{2}\right) \times\left(A_{i}\left(\hat{z}_{i}+2 \sqrt{\hat{z}_{i}^{2}+h^{2}}\right)^{2}-B_{i} h\left(\hat{z}_{i}+2 \sqrt{\hat{z}_{i}^{2}+h^{2}}\right)+C_{i} h^{2}\right)$ and $R_{i}(h)=$ $\left(1+\varsigma_{i}\right)\left(\hat{z}_{i}+2 \sqrt{\hat{z}_{i}^{2}+h^{2}}\right)^{2}-B_{i}^{\prime} h\left(\hat{z}_{i}+2 \sqrt{\hat{z}_{i}^{2}+h^{2}}\right)+C_{i}^{\prime} h^{2}$. Note that, the complexity arises due to the negative sign in $O_{i}(h)$ that makes $\sqrt{O_{i}(h)}$ a complex number. To avoid the negative sign, we rewrite the problem $\mathbf{P 3}^{\prime}$ in the minimization form of sum of ratio of convex functions ${ }^{3}$. Thus, after introducing auxiliary variable $y_{i}$ and applying quadratic transform, P5 becomes a convex problem in $h$ [42]:

$$
\begin{aligned}
& \text { P5 }: \min _{h, y_{i}} \sum_{i=(u, d)} 2 y_{i} \sqrt{O_{i}(h)}-y_{i}^{2}\left(R_{i}(h)\right) \\
& \text { s.t. C1 \& } y_{i} \in \mathbb{R} .
\end{aligned}
$$

For a given $h$, the optimal $y_{i}$ can thus be obtained in closed form as $y_{i}^{\star}=\frac{\sqrt{O_{i}(h)}}{R_{i}(h)}$. The solution to the problem $\mathbf{P 5}$ with $Q_{i}(h)=2 y_{i} \sqrt{O_{i}(h)}-y_{i}^{2} R_{i}(h)$ in the objective function can be obtained using Algorithm 2 that iteratively solves the minimization problem for $h$.

Fig. 5 shows the comparison between the optimal solution obtained from solving (42), (44) and (48), which are represented by blue, black, and red curves, respectively. Evidently, due to the considered approximations of (42), the optimal solution obtained by solving (42) has a slight mismatch with the exact solution obtained by solving (44) using exhaustive search method. However, it is noteworthy that the transformation of (44) into (48) does not impact the optimality of the solution.

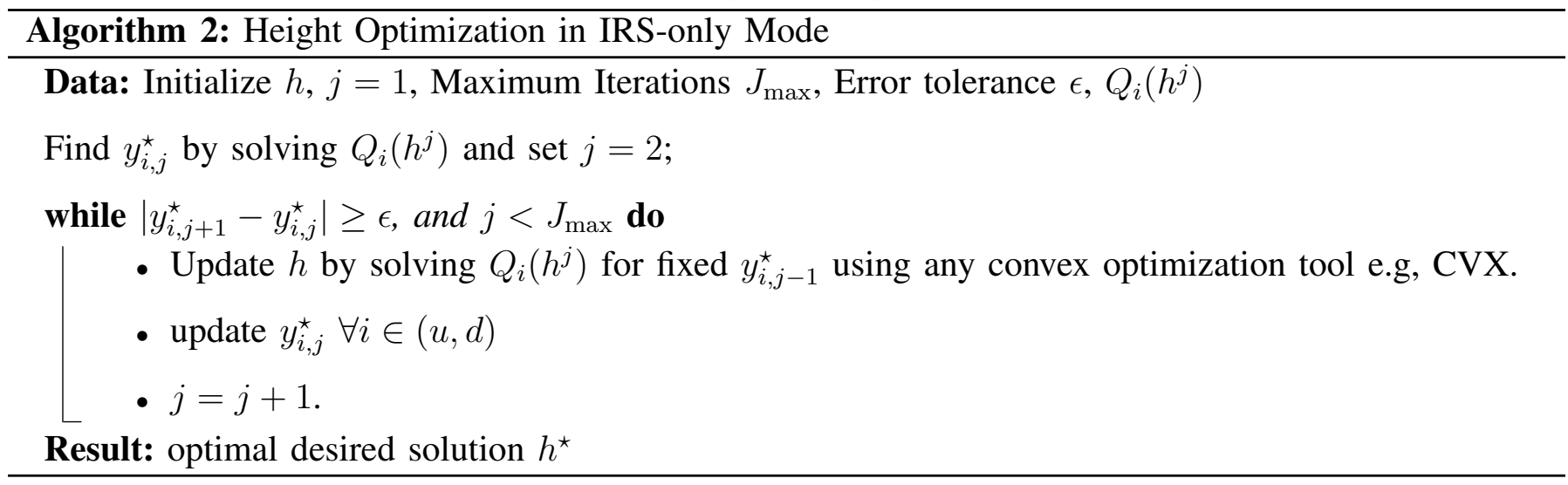

\section{UAV-only Mode: Height Optimization}

We formulate height optimization using (26) which is an approximation of (23) for the UAV-only mode as:

$$
\begin{aligned}
& \mathbf{P 1}: \max _{h} \mathrm{C}_{\mathrm{UAV}}=\operatorname{Blog}_{2}\left(1+\min \left(\mathrm{p}_{\mathrm{u}} \kappa_{\mathrm{u}} \mathrm{d}_{\mathrm{u}}^{-\alpha_{\mathrm{u}}} \Omega_{\mathrm{u}}, \mathrm{p}_{\mathrm{d}} \kappa_{\mathrm{d}} \mathrm{d}_{\mathrm{d}}^{-\alpha_{\mathrm{d}}} \Omega_{\mathrm{d}}\right)\right) \\
& \mathbf{C 1}: h_{\min } \leq h \leq h_{\max } .
\end{aligned}
$$

\footnotetext{
${ }^{3}$ Note that alternation is particularly applicable to the case where quadratic transform P5 is convex, not otherwise.
} 
Using a similar approach followed in (42), i.e. by ignoring logarithm and constant scaling function and considering only the terms that are function of $h$, we recast the optimization problem P1 as follows:

$$
\text { P2 : } \max _{h} \min \left(I_{u} d_{u}^{-\alpha_{u}}, I_{d} d_{d}^{-\alpha_{d}}\right)=\max _{h} \min _{i} I_{i} d_{i}^{-\alpha_{i}}
$$

s.t. C1,

where $I_{u}=p_{u} \kappa_{u} \Omega_{u}, I_{d}=p_{d} \kappa_{d} \Omega_{d}, d_{u}=\sqrt{\hat{z}_{u}^{2}+h^{2}}, d_{d}=\sqrt{\hat{z}_{d}^{2}+h^{2}}$, and $\alpha_{i}$ for $i \in(u, d)$ is given in (43). where $d_{u}$ and $d_{d}$ are convex functions of $h$, whereas $\alpha_{u}$ and $\alpha_{d}$ are ratio of concave and convex functions of $h$. Clearly, this problem is non-convex and cannot be solved directly. Therefore, we take log of $\mathbf{P 2}$ which is an increasing function and does not effect the solution of the original objective. P2 can then be reformulated as follows:

$$
\begin{aligned}
& \mathbf{P} 3: \max _{h} \min _{i} \log \left(I_{i}\right)-\alpha_{i}(h) \log \left(h^{2}+\hat{z}_{i}^{2}\right) \\
& \text { s.t. } \mathbf{C 1}
\end{aligned}
$$

where $\alpha_{i}$ is a ratio of concave and convex functions of $h$, thus the objective function is a ratio of two functions of $h$ for $i \in(u, d)$. However, the ratio in the objective may not necessarily be concave-convex form. However, under a certain condition, we have proved that the objective in $\mathbf{P 3}$ is indeed a concaveconvex form in terms of $h$ ). This guarantees that an optimal solution for P3 can be obtained under specific condition. By substituting $\alpha_{i}(h)$ and simplifying the objective of $\mathbf{P 3}$, we get

$$
O_{i}(h)=G_{1}\left(\hat{z}_{i}+2 \sqrt{\hat{z}_{i}^{2}+h^{2}}\right)^{2}-G_{2} h\left(\hat{z}_{i}+2 \sqrt{\hat{z}_{i}^{2}+h^{2}}\right)+G_{3} h^{2},
$$

where $G_{1}=2 \log \left(I_{i}\right)\left(1+\varsigma_{i}\right)-A_{i} \log \left(h^{2}+\hat{z}_{i}^{2}\right), G_{2}=2 \log \left(I_{i}\right) B_{i}^{\prime}-B_{i} \log \left(h^{2}+\hat{z}_{i}^{2}\right)$, and $G_{3}=2 \log \left(I_{i}\right) C_{i}^{\prime}-$ $C_{i} \log \left(h^{2}+\hat{z}_{i}^{2}\right)$ and denominator function is $R_{i}(h)=\left(1+\varsigma_{i}\right)\left(\hat{z}_{i}+2 \sqrt{\hat{z}_{i}^{2}+h^{2}}\right)^{2}-B_{i}^{\prime} h\left(\hat{z}_{i}+2 \sqrt{\hat{z}_{i}^{2}+h^{2}}\right)+$ $C_{i}^{\prime} h^{2}$. It is straight-forward to see that $R_{i}(h)$ is convex and in the following Proposition, we show that $O_{i}(h)$ in 52 is a concave function of $h$ under a certain condition.

Proposition 5. The $O_{i}(h)$ in (52) is concave when

$$
2 G_{1} \hat{z}_{i}^{3}+\left(4 G_{1}+G_{3}\right)\left(\hat{z}_{i}^{2}+h^{2}\right)^{3 / 2}-G_{2} h\left(3 \hat{z}_{i}^{2}+2 h^{2}\right)
$$

is negative. Using the identity that norm is less than the sum of the sides, i.e. $\sqrt{h^{2}+\hat{z}_{i}^{2}} \leq h+\hat{z}_{i}$, we obtain upper bound on (53) after simplification as

$$
\left(6 G_{1}+G_{3}\right) \hat{z}_{i}^{3}+\left(4 G_{1}+G_{3}-2 G_{2}\right) h^{3}+\left(4 G_{1}+G_{3}\right) \hat{z}_{i} h^{2}+\left(4 G_{1}+G_{3}-3 G_{2}\right) \hat{z}_{i}^{2} h .
$$

Now, for the cases $\hat{z}_{i} \geq h$ and $\hat{z}_{i}<h$ and replacing $\min \left(h, \hat{z}_{i}\right)$ to $\max \left(h, \hat{z}_{i}\right)$ (which gives an upper bound), we obtain the simplified condition for concavity after substituting $G_{1}, G_{2}$ and $G_{3}$ as

$$
\log \left(I_{i}\right) \leq \frac{\left(18 A_{i}-5 B_{i}+4 C_{i}\right)}{36\left(1+\varsigma_{i}\right)-10 B_{i}^{\prime}+8 C_{i}^{\prime}} \log \left(h^{2}+\hat{z}_{i}^{2}\right) .
$$


Now to solve P3, we apply quadratic transformation available for max-min problem [42]. The steps include recasting the problem as maximization of $z$ under the constraint on $h$ such that $z \leq \frac{O_{i}(h)}{R_{i}(h)}$. The constraint $z \leq \frac{O_{i}(h)}{R_{i}(h)}$ can be written using quadratic transform as $2 y_{i} \sqrt{O_{i}(h)}-y_{i}^{2} R_{i}(h) \geq z, \forall i \in(u, d)$ with $y_{i}$ as an auxiliary optimization variable. The equivalent problem of (51) can then be given as:

$$
\begin{aligned}
& \mathbf{P 3}^{\prime}: \max _{h, y_{i}, z} \\
& \mathbf{C} 1 \quad \& \quad \mathbf{C} 2: 2 y_{i} \sqrt{O_{i}(h)}-y_{i}^{2} R_{i}(h) \geq z, \quad \forall i .
\end{aligned}
$$

The above problem cannot be solved due $O_{i}(h)$ being the negative valued function. To solve this, we change $z$ to $-z \geq-\frac{O_{i}(h)}{R_{i}(h)}$ to make $O_{i}(h)$ positive inside the square root in $\mathbf{C 2}$ as follows:

$$
\begin{aligned}
& \mathbf{P} 4: \max _{h, y_{i}, z}-z \\
& \mathbf{C 1} \quad \& \quad \mathbf{C} 2: 2 y_{i} \sqrt{-O_{i}(h)}-y_{i}^{2} R_{i}(h) \geq-z, \quad \forall i
\end{aligned}
$$

Now changing maximization over $h$ to minimization problem as:

$$
\text { P5 : } \min _{h, y_{i}, z} z
$$

$$
\text { C1 \& C2. }
$$

The optimization problem is solved using Algorithm 3 for UAV only mode.

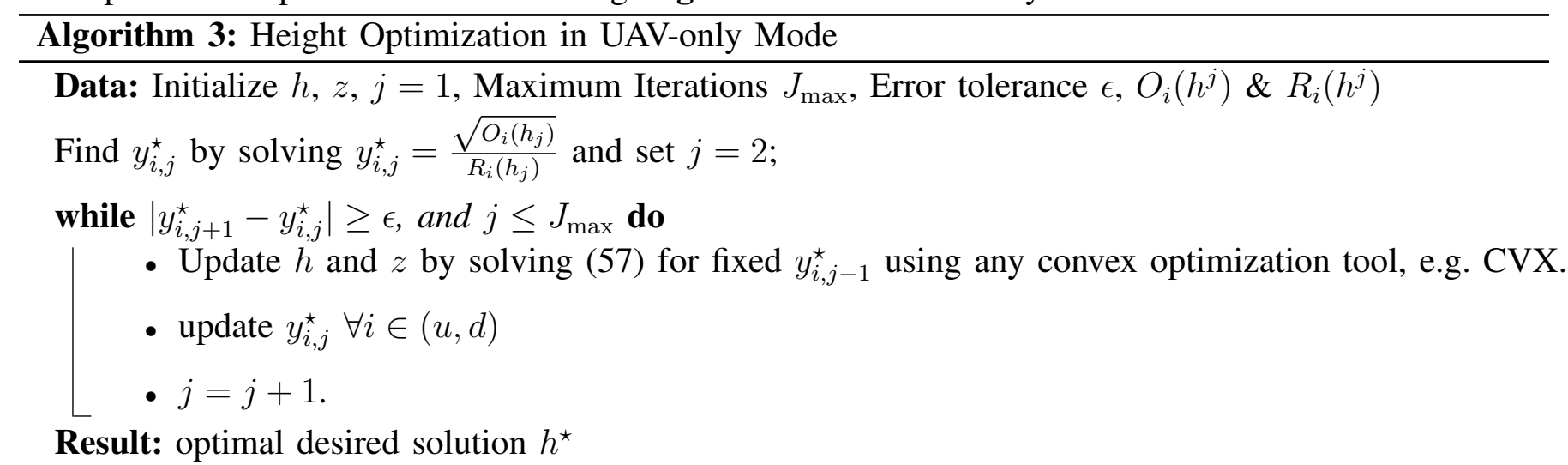

Fig. 6 shows the comparison between the optimal solution obtained from solving (49), (51), and (57), which are represented by blue, black, and red curves, respectively. Clearly, due to the considered approximations the optimal solution obtained by solving (51) has a slight mismatch with the exact solution obtained by solving (49) using exhaustive search method. However, it is noteworthy that the transformation of (51) into (57) does not impact the optimality of the solution.

\section{Mode Selection to Maximize Energy Efficiency}

In this section, we derive the probabilities of selecting modes (UAV-only, IRS-only, integrated UAVIRS) to maximize the energy efficiency. However, first we would like to clarify that the denominator 
(i.e. power consumption) of energy efficiency in integrated UAV-IRS mode will always be higher than the power consumption in UAV-only and IRS-only modes. The reason is that the power consumption of the integrated UAV-IRS mode (the sum of the power consumption of UAV-only and IRS-only modes) is always higher than the power consumption of the UAV-only and IRS-only modes. Furthermore, the numerator which is ergodic capacity in (31) chooses between the maximum SNR of either IRS-only mode or UAV-only mode. As such, the integrated UAV-IRS mode (which is optimal when the objective is to maximize the rate) is not selected when the objective is to maximize energy efficiency. Therefore, the mode selection is essentially performed between UAV-only and IRS-only modes. In what follows, we derive the mode selection probabilities given the instantaneous fading channels and devise a criterion to select how many active IRS elements are needed to maximize energy efficiency in IRS-only mode. We use the proposed criterion for mode selection and obtain optimal heights in above subsections to maximize the overall energy efficiency of the integrated UAV-IRS system.

The probability of selecting IRS-only mode can be formulated as follows:

$$
\mathbb{P}_{\text {IRS }}=\operatorname{Pr}\left(\Gamma_{\text {IRS }} \geq \frac{\Gamma_{\text {UAV }} P_{\text {IRS }}}{P_{\text {UAV }}}\right)=1-\operatorname{Pr}\left(\Gamma_{\text {IRS }}<\frac{\Gamma_{\text {UAV }} \mathrm{P}_{\text {IRS }}}{P_{\mathrm{UAV}}}\right) .
$$

Conditioned on $\Gamma_{\mathrm{UAV}}$, the probability in $(58)$ can be derived as follows:

$$
\mathbb{P}_{\mathrm{IRS}}=\mathbb{E}_{\Gamma_{\mathrm{UAV}}}\left[1-F_{\Gamma_{\mathrm{IRS}}}\left(\frac{\Gamma_{\mathrm{UAV}} P_{I R S}}{P_{\mathrm{UAV}}}\right)\right] \stackrel{(a)}{=} 1-\int_{0}^{\infty} F_{\Gamma_{\mathrm{IRS}}}\left(\frac{\Gamma_{\mathrm{UAV}} P_{I R S}}{P_{\mathrm{UAV}}}\right) f_{\Gamma_{\mathrm{UAV}}}(z) d z,
$$

where $F_{\Gamma_{\mathrm{IRS}}}\left(\frac{\Gamma_{\mathrm{UAV}} P_{\mathrm{IRS}}}{P_{\mathrm{UAV}}}\right)$ is obtained by replacing $\Gamma_{0}$ with $\frac{\Gamma_{\mathrm{UAV}} P_{\mathrm{IRS}}}{P_{\mathrm{UAV}}}$ in (17). The density function of $\Gamma_{\mathrm{UAV}}$ in (6) is obtained by using order statistics and differentiating (11) as $f_{\Gamma_{\mathrm{UAV}}}(z)=\left(1-F_{X_{u}}(z)\right) f_{X_{d}}(z)+$ $\left(1-F_{X_{d}}(z)\right) f_{X_{u}}(z)$, where $f_{X_{i}}(z)$ and $F_{X_{i}}(z)$ are given in (5) and (14), respectively. Subsequently, the probability of UAV-only mode selection can be given as $\mathbb{P}_{\mathrm{UAV}}=1-\mathbb{P}_{\mathrm{IRS}}$.

Now, to maximize the energy efficiency at an arbitrary height, we design the following mode selection criterion based on the average $\mathrm{SNR}^{4}$ to select the IRS-only mode, i.e.

$$
\begin{aligned}
& \mathbb{E}\left[\Gamma_{\mathrm{IRS}}\right] \geq \frac{\mathbb{E}\left[\Gamma_{\mathrm{UAV}}\right] P_{\mathrm{IRS}}}{P_{\mathrm{UAV}}} . \\
& N>\frac{\left(p_{u}-P_{r}(b)+C\right) \min \left(p_{u} \kappa_{u} d_{u}^{-\alpha_{u}} \Omega_{u}, p_{d} \kappa_{d} d_{d}^{-\alpha_{d}} \Omega_{d}\right)-\nu\left(p_{u}+p_{d}+C\right) V d_{u}^{-\alpha_{u}} d_{d}^{-\alpha_{d}}}{\lambda^{\prime}\left(p_{u}+p_{d}+C\right)\left(V d_{u}^{-\alpha_{u}} d_{d}^{-\alpha_{d}}\right)-P_{r}(b) \min \left(p_{u} \kappa_{u} d_{u}^{-\alpha_{u}} \Omega_{u}, p_{d} \kappa_{d} d_{d}^{-\alpha_{d}} \Omega_{d}\right)}-1=N_{\mathrm{th}} .
\end{aligned}
$$

That is, the number of IRS elements should be greater than $N_{\text {th }}$ to enable the IRS-only mode. Another way to maximize the energy efficiency is to calculate $\frac{\mathbb{E}\left[\Gamma_{I R S}\right]}{P_{\mathrm{IRS}}}$ and $\frac{\mathbb{E}\left[\Gamma_{U A V}\right]}{P_{\mathrm{UAV}}}$ with their optimal heights calculated in Section V.B (Algorithm 2) and Section V.C (Algorithm 3), respectively. Then choose the mode and optimal height corresponding to whichever term becomes maximum.

${ }^{4}$ Generally, the instantaneous CSI may not be available at the receiver. 

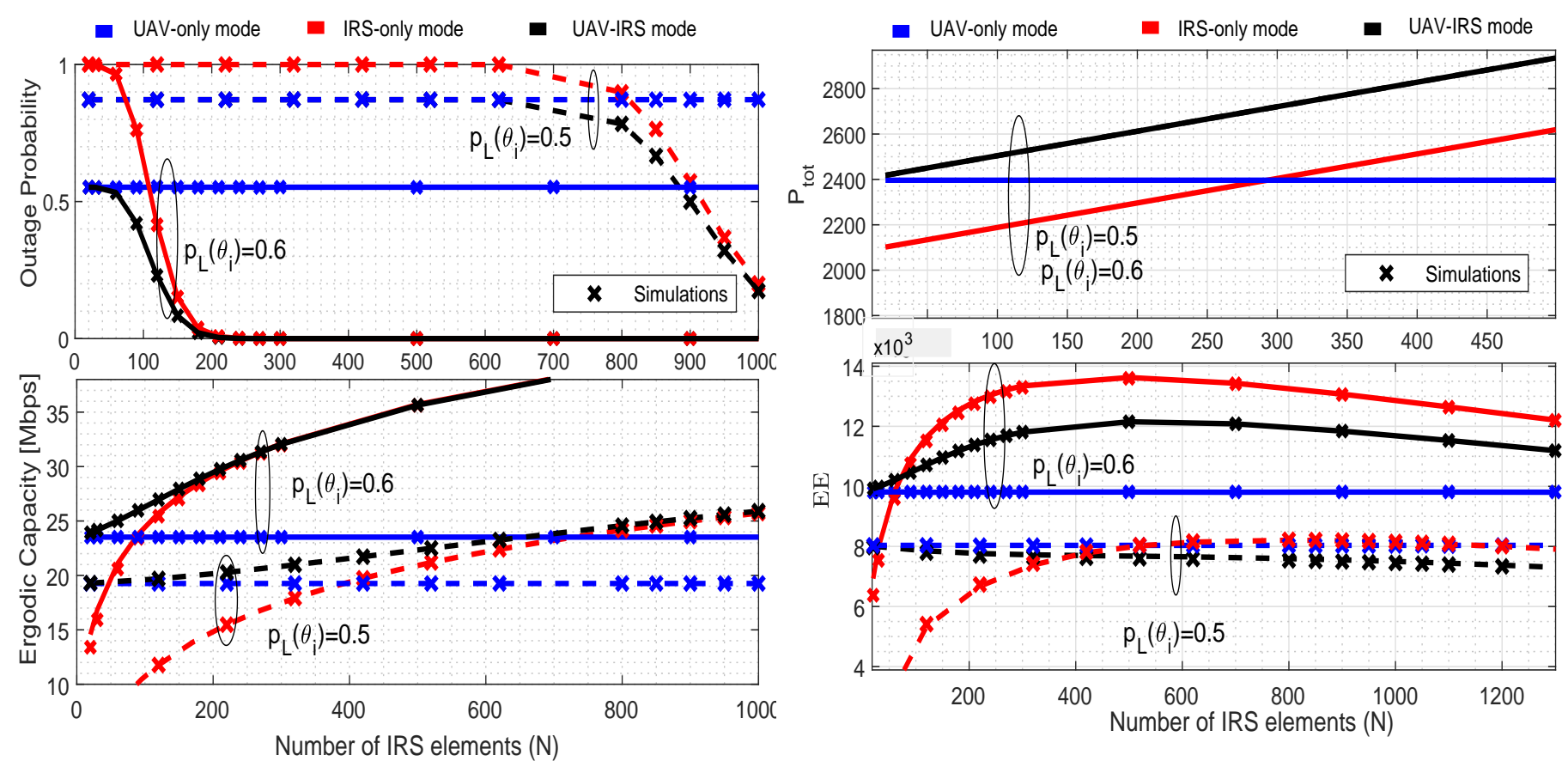

Fig. 7. Performance comparison of outage probability, and ergodic

Fig. 8. Performance comparison of power consumption, and energycapacity for IRS-only, UAV-only and integrated UAV-IRS mode for $d=750 \mathrm{~m}, D=5000 \mathrm{~m}, p_{u}=p_{d}=55 \mathrm{dBm}, P_{r}(b)=108 \times$ $10^{-2} \mathrm{~W}, R_{\mathrm{SI}}=5 \mathrm{~dB}, E_{b} / N_{0}=122 \mathrm{~dB}$, and $\Gamma_{0}=15 \mathrm{~dB}$. efficiency for IRS-only, UAV-only and integrated UAV-IRS mode for $d=750 \mathrm{~m}, D=5000 \mathrm{~m}, p_{u}=p_{d}=55 \mathrm{dBm}, P_{r}(b)=$ $108 \times 10^{-2} \mathrm{~W}, R_{\mathrm{SI}}=5 \mathrm{~dB}, E_{b} / N_{0}=122 \mathrm{~dB}$, and $\Gamma_{0}=15 \mathrm{~dB}$.

Remark: For mode selection based on the power consumption, the integrated UAV-IRS mode will never be selected due to its higher power consumption compared to the UAV-only and IRS-only modes. Furthermore, IRS-only mode will be selected when $N \leq \frac{p_{d}}{P_{r}(b)}$ and vice versa for the UAV-only mode. Similarly, for the SNR-based mode selection, then integrated UAV-IRS mode will always be selected as it chooses the maximum SNR of the IRS-only and UAV-only modes.

\section{NuMERICAL RESUlTS AND DisCUSSION}

In this section, we verify the accuracy of our derived expressions and obtain insights related to the number of IRS elements and the optimal height of UAV for different communication modes. Unless stated otherwise, the simulation parameters are: the maximum distance the UAV can travel $D=2000 \mathrm{~m}$, $B=5 \mathrm{MHz}, H=350 \mathrm{~m}, p_{u}=p_{d}=50 \mathrm{dBm}, \eta_{u}=0.009, \eta_{d}=0.01, \Gamma_{0}=8 \mathrm{~dB}, q_{u}=q_{d}=-1.5$, $v_{u}=v_{d}=3.5, w_{u}=w_{d}=15 \mathrm{~dB}, z_{u}=z_{d}=5, D_{\mathrm{IRS}}=0.5 \mathrm{~m}$. We use $N_{0}=10^{-17} \mathrm{~W} / \mathrm{Hz}$ [44], that justifies the values we use for $\gamma_{u}$ and $\gamma_{d}$ herein.

Fig. 7 compares the outage probability and ergodic capacity w.r.t the number of IRS elements for the UAV-only, IRS-only and integrated UAV-IRS modes. Clearly, UAV-only mode is independent of $N$. 

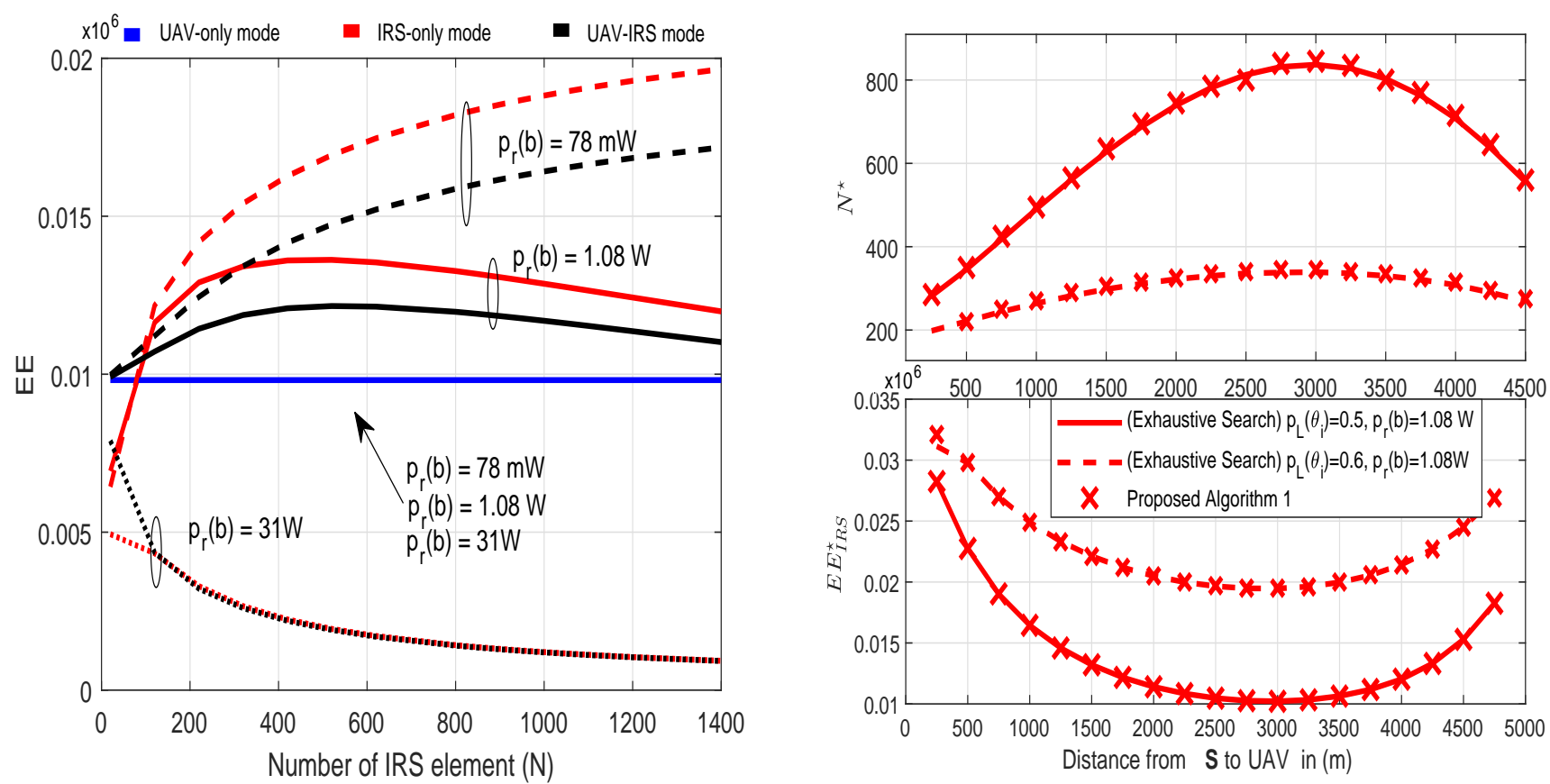

Fig. 9. Power consumption and energy-efficiency w.r.t $N$ for different different bit resolution $P_{r}(b)$ for $d=750 \mathrm{~m}$, and $D=5000 \mathrm{~m}$ $p_{u}=p_{d}=55 \mathrm{dBm}, R_{\mathrm{SI}}=55, E_{b} / N_{0}=122 \mathrm{~dB}$, and $\Gamma_{0}=15 \mathrm{~dB}$.

Fig. 10. Optimal number of IRS elements and the optimal $E E_{\text {IRS }}^{\star}$ comparison for different source to UAV distance for the provided in Fig. 7 and Fig. 8 and different bit resolution $P_{r}(b)$.

However, as $N$ increases, the IRS-only mode and the integrated UAV-IRS mode minimize the outage probability and maximize the capacity due to enhanced IRS transmission link. For larger values of $N$, the IRS-only transmissions become strong and the opportunistic selection between the UAV-only and IRSonly modes improves the performance of integrated UAV-IRS mode. As expected, the integrated UAV-IRS mode outperforms the IRS-only and UAV-only mode for all $N$ in terms of outage and ergodic capacity. An interesting observation is that the lower LoS probability worsens the performance of all schemes. That is, a higher value of $N$ is needed to minimize the outage and maximize the transmission capacity for scenarios with lower LOS.

Fig. 8 compares the power consumption and energy-efficiency w.r.t the number of IRS elements for the UAV-only, IRS-only, and integrated UAV-IRS modes. Clearly, the power consumption and energy efficiency of UAV-only mode do not depend on $N$. However, for the IRS-only mode, the power consumption increases with $N$ and the slope keeps increasing with the value of the power consumption per IRS element $P_{r}(b)$. Note that the power consumption does not change with the LoS probability; therefore the reduction in energy efficiency with the decrease in LoS probability is only due to the reduction in transmission capacity. Furthermore, the energy efficiency first increases up to a certain value of $N$, because the capacity is dominant than power consumption in this regime. Later, for larger values of $N$, the power consumption 

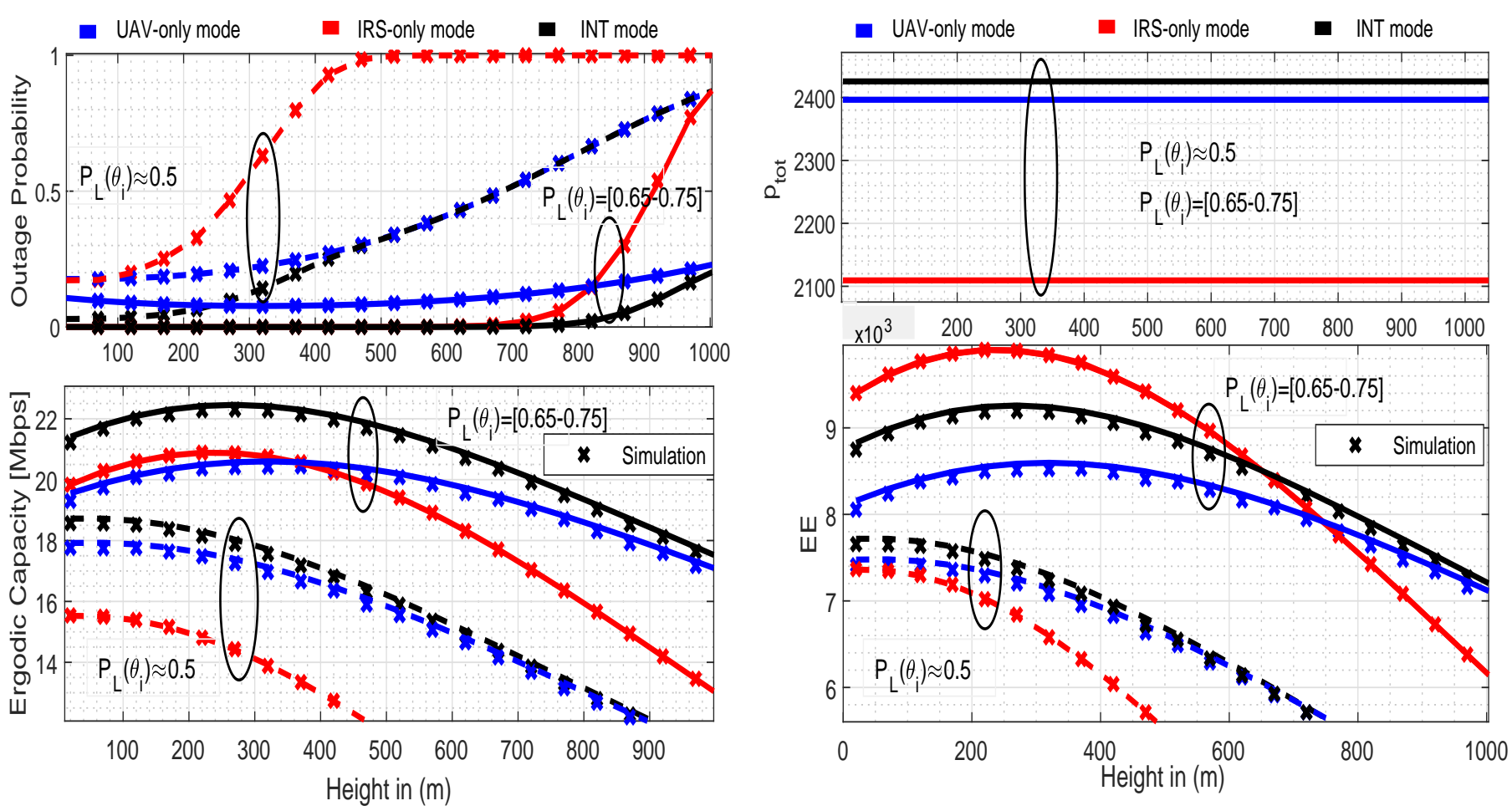

Fig. 12. Performance comparison of power consumption and EE for

Fig. 11. Performance comparison of outage probability and ergodic capacity for IRS-only, UAV-only and integrated UAV-IRS mode for $d=1050 \mathrm{~m}, D=2000 \mathrm{~m}, p_{u}=p_{d}=55 \mathrm{dBm}, N=270$, $P_{r}(b)=108 \mathrm{~mW}, R_{\mathrm{SI}}=45 \mathrm{~dB}$ w.r.t height of UAV. IRS-only, UAV-only and integrated UAV-IRS mode for $d=1050 \mathrm{~m}$, $D=2000 \mathrm{~m}, p_{u}=p_{d}=55 \mathrm{dBm}, N=270, P_{r}(b)=108 m \mathrm{~W}$, $R_{\mathrm{SI}}=45 \mathrm{~dB}$ w.r.t height of UAV.

becomes dominant and thus the reduction in energy efficiency is evident. Finally, it is intuitive to see that the power consumption of the integrated UAV-IRS mode is higher than the other modes; therefore, an efficient mode selection mechanism is important.

Fig. 9 shows the effect of power consumption of bit resolution $P_{r}(b)$ on the energy efficiency of the three communication modes. It is clear that the UAV-only mode is independent of $P_{r}(b)$. However, the IRS-only and integrated UAV-IRS modes show that an optimal number of IRS elements exists which increases with the reduction in $P_{r}(b)$. In particular, for smaller values of $P_{r}(b)$, the EE continues to increase for a wider range of $N$, because the increase in $N$ does not significantly increase the power consumption, whereas the capacity keeps increasing. For higher values of $P_{r}(b)$, the power consumption of IRS elements becomes more dominant than the impact of IRS elements on the ergodic capacity. As such, after a specific value of $N$, a decreasing energy-efficiency trend can be observed. Clearly, for very high values of $P_{r}(b)$, minimizing IRS elements is necessary to maximize energy efficiency. Similar trends are observed for EE in integrated UAV-IRS mode with lower gain than the IRS-only mode, because this mode consumes more power then the IRS-only and UAV-only modes. 


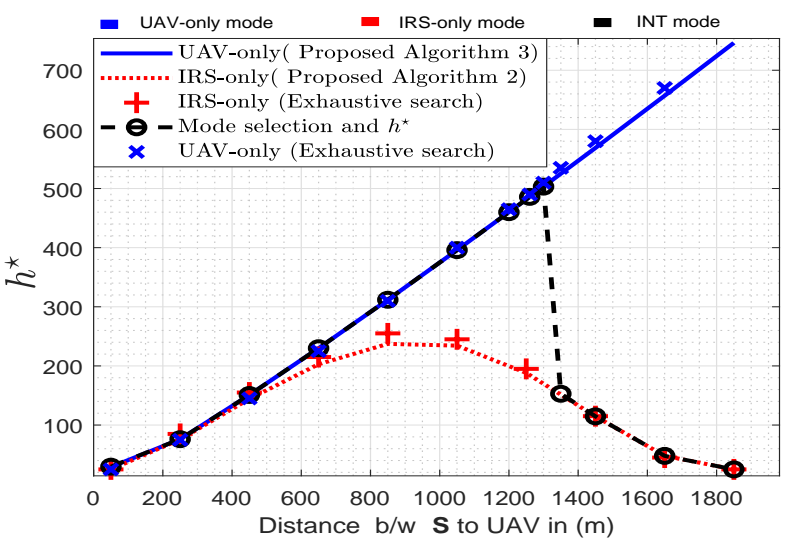

Fig. 13. Optimal UAV height for different source to UAV distance for $E_{b} / N_{0}=130 \mathrm{~dB}, R_{\mathrm{SI}}=38 \mathrm{~dB}, N=30, P_{r}(b)=1.08 \mathrm{~W}$.

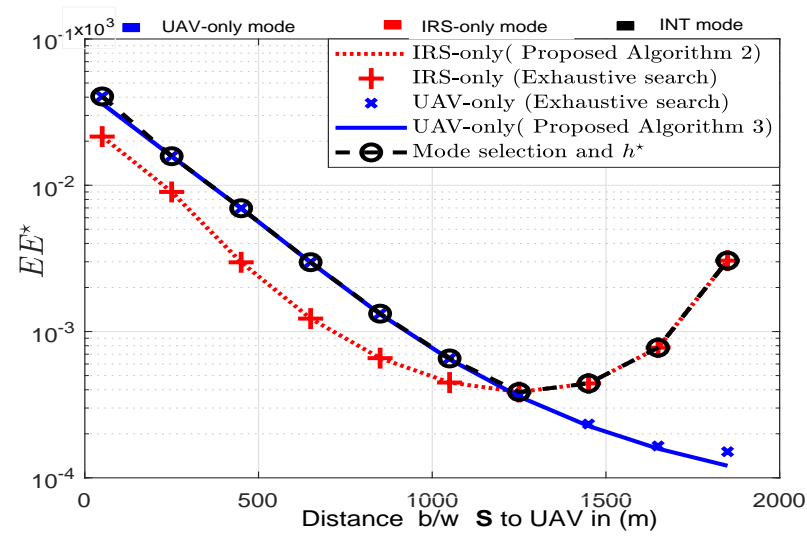

Fig. 14. Optimal EE for different source to UAV distance for $E_{b} / N_{0}=130 \mathrm{~dB}, R_{\mathrm{SI}}=38 \mathrm{~dB}, N=30, P_{r}(b)=1.08 \mathrm{~W}$.

Fig. 10 shows the optimal number of IRS elements $N^{\star}$ (obtained using Algorithm 1) continues to increase as a function of the distance between the source and UAV. However, the corresponding values of optimum energy efficiencies continue to decrease with the increasing distance between the source and UAV. On the other hand, when the distance from the source to UAV decreases, higher values of optimum energy-efficiency can be achieved with less number of IRS elements. This trend is also true when the distance from UAV to destination decreases. The proposed optimal solution (shown by marker) matches well with the optimal solutions obtained by an exhaustive search. Furthermore, we note that a low LoS probability $p_{L}\left(\theta_{i}\right)=0.5$ requires more IRS elements for optimal function while the maximum energy-efficiency values obtained are still low. On the other hand, when $p_{L}\left(\theta_{i}\right)=0.6$, a fewer number of IRS elements provide higher optimum energy efficiency values. In summary, we can conclude that if bit resolution power is very small, then using maximum number of IRS elements is optimal, whereas when the bit resolution power is significantly large, then using minimum number of IRS elements is optimal.

Fig. 11 shows the the outage probability and ergodic capacity versus height of the UAV considering the UAV-only, IRS-only, and integrated UAV-IRS modes. We note that the optimal height varies depending on the selected communication mode. Starting with the outage probability, for weak LoS, we have a higher outage probability in general. However, we note that for weak $\operatorname{LoS} P_{L}\left(\theta_{i}\right) \approx 0.5$, the UAV-only mode outperforms the IRS-only mode, and as expected, the integrated mode performs better than both modes. However, for strong LoS, the IRS-only mode performs better than the UAV-only mode for a wide range of heights. Similar trends can also be seen from the ergodic capacity and that the IRS-only mode dominates the UAV-only mode for smaller heights and the UAV-only mode performs better for higher altitude.

Fig. 12 depicts power consumption and energy efficiency performance with respect to height. The power 
consumption is independent of the LoS probability and height of UAV. The energy-efficiency in strong

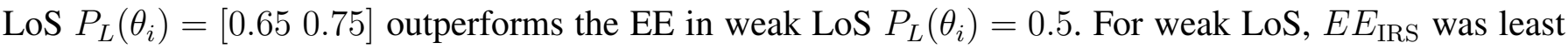
energy-efficient. However, for strong LoS, the IRS-only mode becomes the most energy-efficient mode for a wide range of UAV altitudes, since the IRS only mode power consumption is much lower than the other communication modes (i.e. small $P_{r}(b)$ and $N$ ).

Fig. 13 compares the optimal heights for different distances between source and UAV. This figure shows that the height calculated from the proposed Algorithm 2 and $\mathbf{3}$ matches well with the exact optimal height obtained from exhaustive search. In addition, the performance of analytical mode selection criterion and its corresponding optimal height can also be seen. This shows that for the distance between source and UAV less then $1200 \mathrm{~m}$, the UAV-only mode is optimal, whereas when the UAV is close to the destination the IRS-only mode is optimal. Hence, the optimal height switches to IRS-only height. The same trend is also observed from Figure 14 which represents the optimal energy efficiency vs distance between $\mathbf{S}$ and UAV and follows the same trend as in Fig. 13.

\section{CONCLUSION}

We have analyzed the end-to-end performance in terms of SNR outage probability, ergodic capacity, and energy efficiency for an integrated UAV-IRS relaying system that can operate in three different modes, namely, IRS-only mode, UAV-only mode and integrated UAV-IRS mode. For the IRS-only mode, we optimized the number of IRS elements and UAV height, whereas we have optimized the UAV height for the UAV-only mode. We have observed that the optimal height varies based on the selected transmission mode. We have also provided an analytical criterion for optimal height and mode selection in terms of energy efficiency.

\section{APPENDIX A: RATIO OF CONCAVITY-CONVEXITY OF 44}

We write $-\frac{1}{2} \alpha_{i}(h) \log \left(\hat{z}_{i}^{2}+h^{2}\right)=\frac{-O_{i}(h)}{R_{i}(h)}$. The numerator $-O_{i}(h)$ is concave when the second derivative is $-\frac{d^{2} O_{i}(h)}{d h^{2}} \leq 0$. This is true if,

$$
\begin{aligned}
- & {\left[4 A_{i} \hat{z}_{i}^{5}+\hat{z}_{i}^{4}\left(-2 B_{i}+5 A_{i} \sqrt{\hat{z}_{i}^{2}+h^{2}}\right)+\hat{z}_{i}^{3}\left(8 A_{i} h^{2}-B_{i} \sqrt{\hat{z}_{i}^{2}+h^{2}}\right)+h^{2} \hat{z}_{i}^{2}\left(-4 B_{i}+5\left(3 A_{i}+C_{i}\right) \sqrt{\hat{z}_{i}^{2}+h^{2}}\right)\right.} \\
& +h^{4}\left(-2 B_{i}+3\left(4 A_{i}+C_{i}\right) \sqrt{\hat{z}_{i}^{2}+h^{2}}\right)+\hat{z}_{u}\left(4 A_{i} h^{4}+B_{i} h^{2} \sqrt{\hat{z}_{i}^{2}+h^{2}}\right)+\left(\hat{z}_{i}^{2}+h^{2}\right) \log \left(\hat{z}_{i}^{2}+h^{2}\right) \\
& \left.\times\left(2 A_{i} z_{u s}^{3}+\left(4 A_{i}+C_{i}\right) h^{2} \sqrt{\hat{z}_{i}^{2}+h^{2}}+\hat{z}_{i}^{2}\left(-B_{i}+\left(4 A_{i}+C_{i}\right) \sqrt{\hat{z}_{i}^{2}+h^{2}}\right)\right)\right] \leq 0 .
\end{aligned}
$$


Starting from A.1, we use $\log \left(\hat{z}_{i}^{2}+x^{2}\right) \geq \log \left(\hat{z}_{u}\right)+\frac{x^{2}}{x^{2}+\hat{z}_{i}^{2}}$ and $\log \left(\hat{z}_{i}\right) \geq 1$ when $\hat{z}_{i} \geq 10$ and $\min \left(\hat{z}_{i}\right)>10$ which shows that source and UAV should be at least $10 \mathrm{~m}$ distance apart in the horizontal plane (which gives one of the condition to prove concave numerator). Under this condition, we obtain,

$$
\begin{aligned}
& -\left[6 \hat{z}_{i}^{5} A_{i}+12 A_{i} h^{2} \hat{z}_{i}^{3}+5 h^{4}\left(4 A_{i}+C_{i}\right) \sqrt{\hat{z}_{i}^{2}+h^{2}}+\hat{z}_{i}^{4}\left(9 A_{i}+C_{i}\right) \sqrt{\hat{z}_{i}^{2}+h^{2}}+\hat{z}_{i}^{2} h^{2}\left(27 A_{i}+8 C_{i}\right) \sqrt{\hat{z}_{i}^{2}+h^{2}}+\right. \\
& \left.\hat{z}_{i}\left(4 A_{i} h^{4}+B_{i} h^{2} \sqrt{\hat{z}_{i}^{2}+h^{2}}\right)-\hat{z}_{i}^{3} B_{i} \sqrt{\hat{z}_{i}^{2}+h^{2}}-2 B_{i} h^{4}-3 B_{i} \hat{z}_{i}^{4}-6 B_{i} \hat{z}_{i}^{2} h^{2}\right] \leq 0 .
\end{aligned}
$$

Substituting the lower bound $\sqrt{\hat{z}_{i}^{2}+h^{2}} \geq \max \left(\hat{z}_{i}, h\right)$, which does not change the negativity of the expression, we have

$$
\begin{gathered}
6 \hat{z}_{i}^{5} A_{i}+12 A_{i} h^{2} \hat{z}_{i}^{3}+5 h^{4}\left(4 A_{i}+C_{i}\right) \max \left(\hat{z}_{i}, h\right)+\hat{z}_{i}^{4}\left(\left(9 A_{i}+C_{i}\right) \max \left(\hat{z}_{i}, h\right)\right)+\hat{z}_{i}^{2} h^{2}\left(27 A_{i}+8 C_{i}\right) \times \\
\max \left(\hat{z}_{i}, h\right)+\hat{z}_{i}\left(4 A_{i} h^{4}+B_{i} h^{2} \max \left(\hat{z}_{i}, h\right)\right)-\hat{z}_{i}^{3} B_{i} \max \left(\hat{z}_{i}, h\right)-2 B_{i} h^{4}-3 B_{i} \hat{z}_{i}^{4}-6 B_{i} \hat{z}_{i}^{2} h^{2} \geq 0 .
\end{gathered}
$$

To simplify the expression, we consider case (i) when $\hat{z}_{i}>h$, and substitute $\max \left(\hat{z}_{i}, h\right)=\hat{z}_{i}$ that yields:

$$
\left(15 A_{i}+C_{i}\right) \hat{z}_{i}^{5}+\left(24 A_{i}+5 C_{i}\right) \hat{z}_{i} h^{4}+39 A_{i} h^{2} \hat{z}_{i}^{3}+8 C_{i} h^{2} \hat{z}_{i}^{3}-4 B_{i} \hat{z}_{i}^{4}-5 B_{i} \hat{z}_{i}^{2} h^{2}-2 B_{i} h^{4} \geq 0 .
$$

Replacing $\hat{z}_{i}$ by $h$ in the positive terms and $h$ by $\hat{z}_{i}$ in negative terms, we get

$$
11 B_{i} \hat{z}_{i}^{4}-\left(78 A_{i}+14 C_{i}\right) h^{5}>0 \Longrightarrow z_{i} \geq h^{5 / 4}\left(\frac{78 A_{i}+14 C_{i}}{11 B_{i}}\right)^{1 / 4} .
$$

Similarly, for case (ii): when $h>\hat{z}_{i}$ : we substitute $\max \left(\hat{z}_{i}, h\right) \geq h$ in A.3), replacing $h$ by $\hat{z}_{i}$ in the positive terms and $\hat{z}_{i}$ by $h$ in negative terms and simplification gives

$$
\begin{gathered}
=-\left[6 \hat{z}_{i}^{5} A_{i}+12 A_{i} \hat{z}_{i}^{5}+5 \hat{z}_{i}^{5}\left(4 A_{i}+C_{i}\right)+\hat{z}_{i}^{5}\left(9 A_{i}+C_{i}\right)+\hat{z}_{i}^{5}\left(27 A_{i}+8 C_{i}\right)+4 A_{i} \hat{z}_{i}^{5}+B_{i} \hat{z}_{i}^{4}-12 B_{i} h^{4}\right] \leq 0 \\
\Longrightarrow h \geq \hat{z}_{i}\left(\frac{78 A_{i} \hat{z}_{i}+B_{i}+14 C_{i} \hat{z}_{i}}{12 B_{i}}\right)^{1 / 4}
\end{gathered}
$$

However, the denominator $R_{i}(h)=\left(1+\varsigma_{i}\right)\left(\hat{z}_{i}+2 \sqrt{\hat{z}_{i}^{2}+h^{2}}\right)^{2}-B_{i}^{\prime} h\left(\hat{z}_{i}+2 \sqrt{\hat{z}_{i}^{2}+h^{2}}\right)+C_{i}^{\prime} h^{2}$ is convex when the second derivative of $R_{i}(h)$ is positive. The term $\frac{d^{2} R_{i}(h)}{d h^{2}} \geq 0$ is positive when $2 \hat{z}_{i}^{3} A_{i}+\left(\hat{z}_{i}^{2}+\right.$ $\left.h^{2}\right)\left(4 A_{i}+C_{i}\right) \sqrt{\hat{z}_{i}^{2}+h^{2}} \geq \hat{z}_{i}^{2} B_{i}$ which is true since $2 A_{i} \hat{z}_{i}>B_{i}$ because $A_{i}, B_{i}, C_{i}$ are order of tens but $z_{i}$ is in order of hundreds and thousands, hence $R_{i}(h)$ is convex.

Hence, (A.4) and (A.5) under the constraint $\min \left(\hat{z}_{i}\right)>10$ gives the condition on concavity of $-O_{i}(h)$.

\section{REFERENCES}

[1] Q. Wu and R. Zhang, "Towards smart and reconfigurable environment: Intelligent reflecting surface aided wireless network," IEEE Commun. Mag., vol. 58, no. 1, pp. 106-112, 2020.

[2] C. Huang, A. Zappone, G. C. Alexandropoulos, M. Debbah, and C. Yuen, "Reconfigurable intelligent surfaces for energy efficiency in wireless communication,” IEEE Trans. Commun., vol. 18, no. 8, pp. 4157-4170, 2019.

[3] C. Liaskos, S. Nie, A. Tsioliaridou, A. Pitsillides, S. Ioannidis, and I. Akyildiz, "A new wireless communication paradigm through software-controlled metasurfaces," IEEE Commun. Mag., vol. 56, no. 9, pp. 162-169, 2018. 
[4] Q. Wu and R. Zhang, "Intelligent reflecting surface enhanced wireless network via joint active and passive beamforming," IEEE Trans. Commun., vol. 18, no. 11, pp. 5394-5409, 2019.

[5] E. Vinogradov, H. Sallouha, S. De Bast, M. M. Azari, and S. Pollin, "Tutorial on UAV: A blue sky view on wireless communication," arXiv preprint arXiv:1901.02306, 2019.

[6] M. Mozaffari, W. Saad, M. Bennis, Y.-H. Nam, and M. Debbah, "A tutorial on UAVs for wireless networks: Applications, challenges, and open problems," IEEE Commun. Surveys \& Tutorials, vol. 21, no. 3, pp. 2334-2360, 2019.

[7] B. Ji, Y. Li, B. Zhou, C. Li, K. Song, and H. Wen, "Performance analysis of UAV relay assisted IoT communication network enhanced with energy harvesting," IEEE Access, vol. 7, pp. 38 738-38 747, 2019.

[8] L. Zhou, Z. Yang, S. Zhou, and W. Zhang, "Coverage probability analysis of UAV cellular networks in urban environments," in IEEE Intl. Conf on Commun. Workshops (ICC Workshops), 2018, pp. 1-6.

[9] X. Yuan, Z. Feng, W. Xu, W. Ni, J. A. Zhang, Z. Wei, and R. P. Liu, "Capacity analysis of UAV communications: Cases of random trajectories," IEEE Trans. Vehicular Tech, vol. 67, no. 8, pp. 7564-7576, 2018.

[10] M. Kim and J. Lee, "Outage probability of UAV communications in the presence of interference," in IEEE Global Commun. Conf. (GLOBECOM), 2018, pp. 1-6.

[11] S. Ahmed, A. Mohamed, K. Harras, M. Kholief, and S. Mesbah, "Energy efficient path planning techniques for UAV-based systems with space discretization," in IEEE Wireless Commun. and Netw. Conf., 2016, pp. 1-6.

[12] Y. Zeng and R. Zhang, "Energy-efficient UAV communication with trajectory optimization," IEEE Trans. Wireless Commun., vol. 16, no. 6, pp. 3747-3760, 2017.

[13] D. Yang, Q. Wu, Y. Zeng, and R. Zhang, "Energy tradeoff in ground-to-UAV communication via trajectory design," IEEE Trans. Veh. Technol, vol. 67, no. 7, pp. 6721-6726, 2018.

[14] J. Chakareski, S. Naqvi, N. Mastronarde, J. Xu, F. Afghah, and A. Razi, "An energy efficient framework for UAV-assisted millimeter wave 5G heterogeneous cellular networks," IEEE Trans. Green Commun. Netw., vol. 3, no. 1, pp. 37-44, 2019.

[15] J. Lyu, Y. Zeng, R. Zhang, and T. J. Lim, "Placement optimization of UAV-mounted mobile base stations," IEEE Commun. Letters, vol. 21, no. 3, pp. 604-607, 2017.

[16] M. Alzenad, A. El-Keyi, F. Lagum, and H. Yanikomeroglu, "3-D placement of an unmanned aerial vehicle base station (UAV-BS) for energy-efficient maximal coverage," IEEE Wireless Commun. Letters, vol. 6, no. 4, pp. 434-437, 2017.

[17] J. Lyu, Y. Zeng, and R. Zhang, "Cyclical multiple access in UAV-aided communications: A throughput-delay tradeoff," IEEE Wireless Commun. Letters, vol. 5, no. 6, pp. 600-603, 2016.

[18] T. Shafique, H. Tabassum, and E. Hossain, "End-to-end energy-efficiency and reliability of UAV-assisted wireless data ferrying," IEEE Trans. Commun., vol. 68, no. 3, pp. 1822-1837, 2020.

[19] Z. Yang, C. Pan, K. Wang, and M. Shikh-Bahaei, "Energy efficient resource allocation in UAV-enabled mobile edge computing networks," IEEE Trans. Commun., vol. 18, no. 9, pp. 4576-4589, 2019.

[20] E. Basar, M. Di Renzo, J. De Rosny, M. Debbah, M.-S. Alouini, and R. Zhang, "Wireless communications through reconfigurable intelligent surfaces," IEEE Access, vol. 7, pp. 116753-116773, 2019.

[21] C. Huang, G. C. Alexandropoulos, A. Zappone, M. Debbah, and C. Yuen, "Energy efficient multi-user MISO communication using low resolution large intelligent surfaces," in IEEE Globecom Workshops (GC Wkshps), 2018, pp. 1-6.

[22] E. Björnson, Ö. Özdogan, and E. G. Larsson, "Intelligent reflecting surface vs. decode-and-forward: How large surfaces are needed to beat relaying?" IEEE Wireless Commun. Letters, vol. 9, no. 2, pp. 244-248, 2020.

[23] S. Li, B. Duo, X. Yuan, Y.-C. Liang, and M. Di Renzo, "Reconfigurable intelligent surface assisted UAV communication: Joint trajectory design and passive beamforming," IEEE Wireless Commun. Letters, 2020.

[24] Q. Zhang, W. Saad, and M. Bennis, "Reflections in the sky: Millimeter wave communication with uav-carried intelligent reflectors," arXiv preprint arXiv:1908.03271, 2019. 
[25] R. Méndez-Rial, C. Rusu, N. González-Prelcic, A. Alkhateeb, and R. W. Heath, "Hybrid MIMO architectures for millimeter wave communications: Phase shifters or switches?” IEEE Access, vol. 4, pp. 247-267, 2016.

[26] A. Al-Hourani and K. Gomez, "Modeling cellular-to-UAV path-loss for suburban environments," IEEE Wireless Commun. Letters, vol. 7, no. 1, pp. 82-85, 2018.

[27] M. M. Azari, F. Rosas, K.-C. Chen, and S. Pollin, "Ultra reliable UAV communication using altitude and cooperation diversity," IEEE Trans. Commun., vol. 66, no. 1, pp. 330-344, 2018.

[28] U. Siddique, H. Tabassum, and E. Hossain, "Downlink spectrum allocation for in-band and out-band wireless backhauling of full-duplex small cells," IEEE Trans. Commun., vol. 65, no. 8, pp. 3538-3554, 2017.

[29] D. Bharadia, E. McMilin, and S. Katti, "Full duplex radios," in Proceedings of the ACM Conf. on SIGCOMM, 2013, pp. 375-386.

[30] T. N. Nguyen, P. T. Tran, and M. V. Minh, T.H.Q. Minh, "Two-way half duplex decode and forward relaying network with hardware impairment over Rician fading channel: system performance analysis,” Elektronika Ir Elektrotechnika, vol. 24, no. 2, pp. 74-78, 2018.

[31] A. C. J. Samarasekera, "The performance of dual-hop decode-and-forward underlay cognitive relay networks with interference power constraints over Weibull fading channels," 2014.

[32] Q. Wu and R. Zhang, "Beamforming optimization for intelligent reflecting surface with discrete phase shifts," in IEEE International Conf. on Acoustics, Speech and Signal Processing (ICASSP), 2019, pp. 7830-7833.

[33] J. Lu, S. Wan, X. Chen, and P. Fan, "Energy-efficient 3D UAV-BS placement versus mobile users' density and circuit power," in 2017 IEEE Globecom Workshops (GC Wkshps), 2017, pp. 1-6.

[34] A. Bousia, E. Kartsakli, A. Antonopoulos, L. Alonso, and C. Verikoukis, "Energy efficient schemes for base station management in 4G broadband systems," in Broadband Wireless Access Networks for 4G: Theory, Application, and Experimentation. IGI Global, 2014, pp. 100-120.

[35] M. R. Bhatnagar, "On the capacity of decode-and-forward relaying over Rician fading channels," IEEE Commun. Letters, vol. 17, no. 6, pp. 1100-1103, 2013.

[36] T. Nguyen, T. Quang Minh, P. Tran, and M. Vozňák, "Energy harvesting over Rician fading channel: A performance analysis for half-duplex bidirectional sensor networks under hardware impairments," Sensors, vol. 18, no. 6, p. 1781, 2018.

[37] B. Talha and M. Pätzold, "On the statistical properties of double Rice channels," in Proc. 10th Intr. Symposium on Wireless Personal Multimedia Commun., WPMC, 2007, pp. 517-522.

[38] J. Salo, H. M. El-Sallabi, and P. Vainikainen, "The distribution of the product of independent Rayleigh random variables," IEEE Trans. Antennas Propag., vol. 54, no. 2, pp. 639-643, 2006.

[39] H. Tabassum and E. Hossain, "Coverage and rate analysis for co-existing RF/VLC downlink cellular networks," IEEE Trans. Commun., vol. 17, no. 4, pp. 2588-2601, 2018.

[40] F. Jiang and A. L. Swindlehurst, "Dynamic UAV relay positioning for the ground-to-air uplink," in IEEE Globecom Workshops, 2010, pp. 1766-1770.

[41] J. Proakis and M. Salehi, Digital communications. McGraw-Hill, 2007.

[42] K. Shen and W. Yu, "Fractional programming for communication systems- Part I: Power control and beamforming," IEEE Transactions on Signal Processing, vol. 66, no. 10, pp. 2616-2630, 2018.

[43] A. Zappone, E. Björnson, L. Sanguinetti, and E. A. Jorswieck, "Achieving global optimality for energy efficiency maximization in wireless networks," IEEE Trans. Signal Process., 2016.

[44] S. Sekander, H. Tabassum, and E. Hossain, "Multi-tier drone architecture for 5G/B5G cellular networks: Challenges, trends, and prospects," IEEE Commun. Mag., vol. 56, no. 3, pp. 96-103, 2018. 